\title{
A radio continuum study of the Magellanic Clouds
}

\author{
VII. Discrete radio sources in the Magellanic Clouds ${ }^{\star}$ \\ M.D. Filipovic ${ }^{1,2,3}$, R.F. Haynes ${ }^{3,1}$, G.L. White ${ }^{1}$, and P.A. Jones ${ }^{1}$ \\ 1 University of Western Sydney, Nepean, P.O. Box 10, Kingswood, NSW 2747, Australia \\ e-mail: M.Filipovic@uws.edu.au; p.jones@nepean.uws.edu.au; g.white@nepean.uws.edu.au \\ 2 Max-Planck-Institut für extraterrestrische Physik, Giessenbachstraße, D-85740 Garching, Germany \\ 3 Australia Telescope National Facility, CSIRO, P.O. Box 76, Epping, NSW 2121, Australia \\ e-mail:mfilipovic@atnf.csiro.au; rhaynes@atnf.csiro.au
}

Received November 28; accepted December 24, 1997

\begin{abstract}
We present a study of discrete radio sources in the Magellanic Clouds (MCs) using the latest large-scale radio surveys made with the Parkes radio telescope between 1.4 and $8.55 \mathrm{GHz}$. These surveys achieved higher sensitivity then previous surveys done with the Parkes telescope and so the number of discrete radio sources detected towards the MCs has increased by factor of five. Also, we have obtained improved positions, flux densities and radio spectral indices for all of these sources.

A total of 483 sources towards the Large Magellanic Cloud (LMC) and 224 towards the Small Magellanic Cloud (SMC) have been detected at at least one radio frequency. Most of the MC's sources have been classified in one of three groups: SNRs, H II regions or background sources according to classification criteria established here. In total, 209 discrete radio sources in the LMC and the 37 sources in the SMC are classified here to be either H II regions or SNRs. We investigate their luminosity functions as well as the statistics of background sources behind the MCs. Also, we examine the distribution of SNRs and HII regions in the MCs.
\end{abstract}

Key words: galaxies: Magellanic Clouds - radio continuum: galaxies — ISM: HII regions — ISM: supernova remnants (SNRs) — galaxies: ISM

Send offprint requests to: M.D. Filipović

* Tables 5 and 6 are only available electronically at the CDS via ftp 130.79 .128 .5 or via http://cdsweb.ustrasbg.fr/Abstract.html

\section{Radio-continuum surveys of the Magellanic Clouds}

During the last few decades, the Magellanic Clouds (MCs) have been investigated over almost all of the electromagnetic spectrum. Radio-continuum investigations of the MCs started with the detection by Mills \& Little (1953). Since that time, numerous radio surveys have been undertaken. These include: Mills (1955) at 85.5 MHz; Mills \& Little (1959) at $96.8 \mathrm{MHz}$ and $158 \mathrm{MHz}$; Shain (1959) at $19.7 \mathrm{MHz}$; Mathewson \& Healey (1964a) at $1400 \mathrm{MHz}$; Alvarez et al. (1987) at $45 \mathrm{MHz}$; and Mountfort et al. (1987) at $2300 \mathrm{MHz}$. The low-resolution observations helped to determine the large-scale structure and the total radio spectrum of the MCs (Mills \& Little 1959; Haynes et al. 1991; Klein et al. 1991) whereas high-resolution studies concentrated on discrete sources in the Clouds.

\subsection{High-frequency radio continuum surveys of discrete sources in the Magellanic Clouds}

The first high-frequency radio-continuum detection of discrete sources in the MCs was made by Mathewson \& Healey (1964b) at $1.4 \mathrm{GHz}$. In the past 30 years, numerous radio observations of many discrete sources in the MCs have been undertaken.

In 1972, McGee et al. (1972a) made detailed observations of the $\mathrm{LMC}$ at $5.00 \mathrm{GHz}$ with the Parkes radio telescope (See Table 1 for a summary of the radio-source surveys of the LMC) and established the first catalogue of discrete radio sources in the LMC, known as the MC catalogue. Later, McGee et al. (1976) presented a list of sources towards the Small Magellanic Cloud (SMC) at $5.00 \mathrm{GHz}$ (See Table 2 for a summary of the radio-source surveys of the SMC). McGee et al. (1972b) and Bolton \& Butler (1975) observed the MCs at $2.70 \mathrm{GHz}$ which became part of the PKSCAT-90 catalogue (Otrupcek \& Wright 1991). 
Table 1. Summary of the previous high-frequency radio surveys of the LMC

\begin{tabular}{llccl}
\hline \hline Instrument & $\begin{array}{c}\text { Frequency } \\
(\mathrm{GHz})\end{array}$ & $\begin{array}{c}\text { Beam Size } \\
\text { (arcmin) }\end{array}$ & $\begin{array}{c}\text { No. of } \\
\text { Sources } \\
\text { Detected }\end{array}$ \\
\hline Molonglo & 0.408 & $2.62 \times 2.86$ & 227 & Clarke et al. 1976 \\
MOST & 0.843 & 0.75 & 42 & Mills et al. 1984a,b \\
Parkes & 2.70 & 7.7 & 38 & McGee et al. 1972b \\
Parkes & 4.85 & 4.9 & 390 & Wright et al. 1994 \\
Parkes & 5.009 & 4.0 & 95 & McGee et al. 1972a \\
Parkes & 8.80 & 2.5 & 35 & McGee et al. 1978 \\
Parkes & 14.70 & 2.2 & 33 & Milne et al. 1980 \\
\hline
\end{tabular}

Table 2. Summary of the previous high-frequency radio surveys of the SMC

\begin{tabular}{llccl}
\hline \hline Instrument & $\begin{array}{c}\text { Frequency } \\
(\mathrm{GHz})\end{array}$ & $\begin{array}{c}\text { Beam Size } \\
(\text { arcmin })\end{array}$ & $\begin{array}{c}\text { No. of } \\
\text { Sources } \\
\text { Detected }\end{array}$ \\
\hline Molonglo & 0.408 & $2.62 \times 2.86$ & 75 & Clarke et al. 1976 \\
MOST & 0.843 & 0.75 & $\sim 1500$ & Ye 1988, 1993 \\
Parkes & 1.40 & 15.0 & 21 & McGee et al. 1976 \\
Parkes & 2.70 & 7.7 & 25 & PKSCAT-90 \\
Parkes & 4.85 & 4.9 & 194 & Wright et al. 1994 \\
Parkes & 5.009 & 4.0 & 27 & McGee et al. 1976 \\
Parkes & 8.80 & 2.5 & 13 & McGee et al. 1976 \\
\hline
\end{tabular}

After Mills \& Aller 1971 detected a few sources at $0.408 \mathrm{GHz}$, as a part of a whole sky survey, Clarke et al. (1976) observed the MCs with the Molonglo radio telescope and produced the MC4 catalogue of 227 sources towards the LMC and 75 towards the SMC. Also, some sources in the direction of the MCs (mainly background) appear in the MRC catalogue of Large et al. (1981).

McGee et al. (1976) and McGee et al. (1978) investigated the MCs with the Parkes telescope at $8.80 \mathrm{GHz}$. The PKSCAT-90 compilation (Otrupcek \& Wright 1991) contains a number of MC sources with fluxes at 1.40, 2.70, 5.00 and $8.40 \mathrm{GHz}$.

New techniques and equipment evolved in the early 1980s, and with improved sensitivity, increased the interest in discrete sources in the MCs, such as $\mathrm{HII}$ regions and supernova remnants (SNRs). Milne et al. (1980) observed a number of interesting LMC sources at $14.70 \mathrm{GHz}$ to estimate their radio spectral index. Mills (1983), Mills et al. (1984a,b), Ye (1988) and Ye \& Turtle (1993) observed selected fields in the MCs at $0.843 \mathrm{GHz}$ with the Molonglo Synthesis Telescope (MOST), and Sabalisck \& Abraham (1991) observed the 30 Doradus region at $22 \mathrm{GHz}$. Recently, Wright et al. (1994) surveyed the MCs as part of the PMN southern sky survey at $4.85 \mathrm{GHz}$.

Following the first radio classification of sources in the LMC based on studies of the spectral index (McGee \& Newton 1972), Milne et al. (1980) and Mathewson et al. (1983,a, 1984, 1985) provided the first detailed analysis of the spectra of some 40 sources, most of which were SNRs. The radio spectral index study of the SMC sources was undertaken by Loiseau et al. (1987) for some 28 sources.

\subsection{Other Surveys of discrete sources in the Magellanic Clouds}

Numerous observations of the MCs in the optical, $\mathrm{H} \alpha$, infrared (IR), far- infrared (FIR), and X-ray wavelengths have been undertaken together with the multi-waveband cross-correlation of the discrete sources.

Optical observations of the MCs made by Henize (1956) resulted in the first catalogue of emission-line objects. Hodge \& Wright (1967) presented an optical atlas of the LMC and, 10 years later, of the SMC (Hodge \& Wright 1977). Photographic H $\alpha$ emission-line surveys by Henize (1956; N catalogue) and by Davies et al. (1976; DEM catalogue) have provided identifications, positions and sizes for several hundred sources in the two galaxies, ranging in scale from unresolved compact emission regions to the giant 30 Doradus complex in the LMC. Subsequent photometric surveys have provided quantitative information on the resolved MCs sources (Caplan \& Deharveng 1985; Kennicutt \& Hodge 1986; Bothun \& Thompson 1988; Copetti \& Dottori 1989; le Coarer et al. 1993; Caplan et al. 1996). Also some [S II] and [O III] line surveys for detection of specific sources in the MCs, such as SNRs, were made by Mathewson et al. (1983, 1984, 1985). A number of MC objects appear in Sinnott's (1988) NGC (IC) catalogue of nebulae.

Lucke \& Hodge (1970) published a list of 122 stellar associations in the LMC and Hodge (1985) a list of 70 stellar associations in the SMC. Up to 60 star clusters have been identified in the SMC and some 1600 in the LMC. Sanduleak et al. (1978) and Sanduleak (1984) published a list of planetary nebulae (PN), and Morgan (1994, 1995) updated these lists with 265 and $62 \mathrm{PN}$ in the LMC and SMC, respectively.

The X-ray surveys of the LMC have been presented in Long et al. (1981; LHG catalogue) and in Wang et al. (1991; W catalogue). The SMC X-ray surveys can be found in Seward \& Mitchell (1981; 1E catalogue), Inoue et al. (1983; IKT Catalogue), Bruhweiler et al. (1987) and in Wang \& Wu (1992). All these surveys are based on observations made with the Einstein satellite. Almost half of the X-ray sources in the LMC field catalogued by Wang et al. (1991) were confirmed as SNRs, H II regions or X-ray binaries. The other half are foreground stars or background objects (e.g. clusters of galaxies, radio galaxies, quasars). In the Wang \& Wu (1992) survey, out of 70 sources towards the SMC, 24 are intrinsic. Trümper et al. (1991), Pietsch \& Kahabka (1993), Snowden \& Petre (1994), Kahabka \& Pietsch (1993) and Schmidtke et al. (1994) also made X- ray observations of the MCs with the ROSAT satellite. In addition, X-ray ROSAT All Sky Survey (RASS) results are available from Pietsch et al. (in preparation) and Kahabka et al. (in preparation). The ASCA satellite X-ray observations of some LMC SNRs can be found in Hughes et al. (1995). 
Vacuum ultraviolet (VUV) imagery of the LMC was obtained with an electrographic Schmidt camera during the Black Brant rocket mission in 1977 (Smith et al. 1987 and references therein) and the far-ultraviolet (FUV) survey with Apollo 16 mission (Page \& Carruthers 1978). These surveys resulted in the discovery of a number of star-forming regions in the LMC. The SMC was surveyed with the ultraviolet imaging telescope (UIT) during the Astro-1 mission (December 1990) and the Astro-2 mission (March 1995) of space shuttle Endeavor (Cornett et al. 1994; 1997).

The IRAS catalogue of sources in the MCs at four infrared frequencies was published by Schwering (1989) and Schwering \& Israel $(1989,1990)$. A complete CO millimetre-wave survey of the MCs was presented by Cohen et al. (1988), Israel et al. (1993) and Rubio et al. (1991, 1996). Following these millimetre- wave surveys, the first high-resolution SEST observations of some compact $\mathrm{H}$ II regions in the MCs are presented in Rubio et al. (1993), Garay et al. (1993), Kutner et al. (1997), Chin et al. $(1996,1997)$ and in Hunt (in preparation).

\subsection{New-generation surveys of discrete radio sources in the Magellanic Clouds}

Parkes radio surveys by Haynes et al. (1991), Filipović et al. (1995; hereafter Paper IV), Filipović et al. (1996, hereafter Paper IVa) and Filipović et al. (1997, hereafter Paper V) of the MCs are now available.

There are several other high-resolution radio surveys of the MCs in progress. Continuum surveys at 1.40 and $2.30 \mathrm{GHz}$ of the MCs have been made with the Australia Telescope Compact Array (ATCA; L. StaveleySmith et al. in preparation). The SMC H I observations with the ATCA have been published by Staveley-Smith et al. $(1995,1997)$ and some results of the LMC H I surveys can be found in Kim et al. (1997). Complete MOST surveys at $843 \mathrm{MHz}$ of both Clouds are in preparation but some results were presented in Mills et al. (1984b), Ye (1988) and Ye \& Turtle (1993).

In this paper we will present all sources found in the new Parkes radio surveys together with their fluxes and radio spectral indices. Also, we classify each source as either an HII region, SNR or background object. In Sect. 2 we discuss briefly the Parkes radio surveys of discrete sources towards the MCs. In Sects. 3 and 4 we analyse, discuss and classify all discrete sources in the observed field of the MCs. We investigate the intrinsic source luminosity function in Sect. 5 and in Sect. 6 we discuss the source distribution throughout the MCs.

\section{New Parkes radio surveys}

In 1986, an international collaboration was launched using the Parkes 64-m telescope to survey the MCs at several
Table 3. Parameters of the six radio surveys of the LMC

\begin{tabular}{ccccc}
\hline \hline $\begin{array}{c}\text { Frequency } \\
(\mathrm{GHz})\end{array}$ & $\begin{array}{c}\text { Beam } \\
\text { Size } \\
\text { (arcmin) }\end{array}$ & $\begin{array}{c}\text { Field } \\
\text { Size } \\
(\text { Degree })\end{array}$ & $\begin{array}{c}\text { rms } \\
\text { Noise } \\
\left(\mathrm{mJy}_{\text {beam }}{ }^{-1}\right)\end{array}$ & $\begin{array}{c}\text { No. of } \\
\text { Sources } \\
\text { Detected }\end{array}$ \\
\hline 1.40 & 15.2 & $10^{\circ} \times 10^{\circ}$ & 30 & 192 \\
2.30 & 8.9 & $10^{\circ} \times 10^{\circ}$ & 30 & 119 \\
2.45 & 8.9 & $10^{\circ} \times 10^{\circ}$ & 15 & 338 \\
4.75 & 4.8 & $8^{\circ} \times 9^{\circ}$ & 8 & 373 \\
4.85 & 4.9 & $10^{\circ} \times 10^{\circ}$ & 5 & 332 \\
8.55 & 2.7 & $7^{\circ} \times 6^{\circ}$ & 9 & 212 \\
\hline
\end{tabular}

Table 4. Parameters of the five radio surveys of the SMC

\begin{tabular}{ccccc}
\hline \hline $\begin{array}{c}\text { Frequency } \\
(\mathrm{GHz})\end{array}$ & $\begin{array}{c}\text { Beam } \\
\text { Size } \\
(\text { arcmin })\end{array}$ & $\begin{array}{c}\text { Field } \\
\text { Size } \\
(\text { Degree })\end{array}$ & $\begin{array}{c}\text { rms } \\
\text { Noise } \\
\left(\text { mJy beam }^{-1}\right)\end{array}$ & $\begin{array}{c}\text { No. of } \\
\text { Sources } \\
\text { Detected }\end{array}$ \\
\hline 1.42 & 13.8 & $7.5^{\circ} \times 7.5^{\circ}$ & 15 & 85 \\
2.45 & 9.0 & $7^{\circ} \times 7^{\circ}$ & 10 & 107 \\
4.75 & 4.5 & $6.5^{\circ} \times 4.5^{\circ}$ & 6 & 99 \\
4.85 & 4.9 & $7.5^{\circ} \times 7.5^{\circ}$ & 5 & 187 \\
8.55 & 2.7 & $4^{\circ} \times 3^{\circ}$ & 10 & 41 \\
\hline
\end{tabular}

radio frequencies with moderate angular resolution using a full polarization system (Haynes et al. 1986; Haynes et al. 1991). Between 1988 and 1991, four large-scale surveys of the MCs were undertaken by a team from the Australia Telescope National Facility (ATNF) and the Max-PlanckInstitut für Radioastronomie, Bonn (MPIfR).

To date, the project has resulted in several studies which describe the surveys (Haynes et al. 1986, 1991), the FIR/radio correlation ( $\mathrm{Xu}$ et al. 1992), thermal and non-thermal components (Klein et al. 1989, 1991), magnetic field in the MCs (Klein et al. 1993), the radio source catalogues at six frequencies (Papers IV, IVa and V), the discrete source radio/X-ray correlation (Filipović et al. 1998a, hereafter Paper VI) and the discrete source radio/IR correlation (Filipović et al. 1998b, hereafter Paper VIII).

The search for discrete radio sources in the MCs has resulted in catalogues at $1.40(1.42 \mathrm{GHz}$ for the SMC), $2.45,4.75$ and $8.55 \mathrm{GHz}$ at resolutions of 15.2 (13.8), 8.85, 4.8 and $2.7^{\prime}$ (HPBW), respectively (for details see Table 3 and Table 4). In addition to these specific surveys, a part of the Parkes-MIT- NRAO (PMN) southern all-sky survey at $4.85 \mathrm{GHz}$ by Wright et al. (1994) covered the field of the MCs.

The catalogues of the LMC radio sources at these five frequencies were published in Paper IV. An additional survey and catalogue of the LMC sources at $2.30 \mathrm{GHz}$ appears in Paper IVa. The SMC sources are catalogued in Paper V.

Tables 3 and 4 list the survey material of the LMC and the SMC that is used in this study of discrete sources in the MCs. The first column in Tables 3 and 4 shows the 
observing frequency; the second shows the resolution ${ }^{1}$; the third shows the size of the observed field; the fourth gives an estimate of the noise in the maps and the fifth gives the number of sources detected at the particular frequency.

\section{Source radio spectral index analysis}

Multi-frequency analysis gives a far better understanding of the nature of discrete sources. The MCs are essentially transparent to radio emission, and all catalogues of MC sources contain not only sources located within the Clouds, but also background sources lying behind and seen through the Clouds. With this in mind, sources in the direction of the Clouds may be divided into three groups:

1. non-thermal sources, such as:

a) background quasars and galaxies

b) SNRs in the Clouds and

2. thermal sources, such as H II regions in the Clouds.

This paper uses the results of the six radio surveys to deduce the spectral index for each object and then uses the spectral index estimate in a "first-pass" classification of each source into these three categories. The uncertainties in the observed flux densities lead to the uncertainties in the estimated spectral index and subsequently to uncertainties in classification. The uncertainties and the calibration of the flux-density scale for the our six surveys $(1.40,2.30,2.45,4.75,4.85$ and $8.85 \mathrm{GHz})$ are discussed in Papers IV, IVa and V.

\subsection{Estimating the spectral index}

Estimates of the spectral index $(\alpha)$ of each radio source are based on flux densities obtained from our survey results (Papers IV, IVa and V) and from a range of radiofrequency catalogues that have been published (Tables 1 , 2,3 and 4). The spectral index $\alpha$ is defined by the relation $S_{\nu} \sim \nu^{\alpha}$, where $S_{\nu}$ is the integrated flux density and $\nu$ is frequency.

The integrated flux densities at the various frequencies were plotted as $\log \left(S_{\nu}\right)$ versus $\log \left(\nu_{\mathrm{GHz}}\right)$. For 422 radio sources in the field of the LMC (Table 5), straight lines were fitted with a simple linear regression to produce the best estimates of spectral index. For 162 sources in the field of the SMC (out of 224) we estimated the spectral index (Table 6).

For some sources a simple power-law spectrum could not be applied and these sources were examined individually (two-point spectral indices have been computed) and remarked on in Cols. 19 and 16 of Tables 5 and 6 , respectively. No spectral index was calculated for $61 \mathrm{LMC}$ and 62

\footnotetext{
${ }^{1}$ Nominal beam sizes are obtained from the header of FITS file. Effective beam sizes are discussed in Papers IV, IVa and V.
}

SMC sources as these sources were seen either only at the two closely-spaced frequencies of $4.75 \mathrm{GHz}$ and $4.85 \mathrm{GHz}$ (20 sources for the LMC and 5 for the SMC) or at only one of the Parkes radio frequencies (41 towards the LMC and 57 towards the SMC). The errors in spectral index $(\Delta \alpha)$ have been deduced from the linear-regression uncertainty, given the scatter in flux density.

Tables 5 and 6 gives the source names which have been adopted from Papers IV and V (Col. 1), the flux density at each radio frequency (Cols. 2-15 for the Table 5 and Cols. 2-12 for the Table 6), the spectral index $\alpha$ and uncertainty $\Delta \alpha$ (Cols. 17 for the Table 5 and Col. 14 for the Table 6). Columns 18 (Table 5) and 15 (Table 6) list, where known, the published source type (H II region, SNR or background). Note that capitals (BG, SNR and $\mathrm{H}$ II) are used for classifications from previous works, and lower case (bg, snr and hii) for sources classified here. The question-mark indicates probable but not certain classification. Columns 19 (Table 5) and 16 (Table 6) give the reference for this source designation. Columns 20 (Table 5) and 17 (Table 6) give some comments, where SB (X) stands for "X-ray bright superbubble" and SGS stands for "supergiant shell" (Chu et al. 1994). Columns 21 (Table 5) and 18 (Table 6 ) contain the spectral index from previous work.

Tables 5 and 6 include sources observed over a range of wavelengths - X-ray, $\mathrm{H} \alpha$, optical, infrared and radio continuum. Of the 483 radio sources catalogued towards the LMC and the 224 towards the SMC, we have compared and identified sources with various ranges of catalogues (for details see Table 7). These identifications have been included in Papers IV, IVa and V (Table 7).

\subsection{Comparison with other published spectral indices}

Spectral indices for 74 "well-known" sources towards the LMC and for 46 sources towards the SMC were compared with spectral indices for the same sources previously published (McGee \& Newton 1972; McGee et al. 1976; Milne et al. 1980; Mills et al. 1984b; Mathewson et al. 1983, 1984, 1985; Loiseau et al. 1987).

Figures $1 \mathrm{a}$ and $1 \mathrm{~b}$ show the correlation between the results of previous work and those estimated here. There is an obvious agreement between the two determinations of spectral index, with the difference ("old" - "new") $=0.03 \pm 0.02$ for the LMC and $-0.07 \pm 0.04$ for the SMC. The standard deviation of the difference is 0.17 for the LMC and 0.27 for the SMC.

\subsection{Source radio spectral-index classification}

McGee \& Newton (1972) divided all radio sources towards the LMC into three groups, based on their radio spectrum, as follows: 
Table 7. Identification of Parkes radio sources with other MCs catalogues

\begin{tabular}{|c|c|c|c|c|c|}
\hline $\begin{array}{l}\text { Catalogue } \\
\text { Name }\end{array}$ & Galaxy & Frequency & $\begin{array}{c}\text { No. of } \\
\text { Sources in } \\
\text { Catalogue }\end{array}$ & $\begin{array}{l}\text { No. of Sources } \\
\text { common to our } \\
\text { radio survey }\end{array}$ & Reference \\
\hline $\mathrm{N}$ & $\mathrm{LMC}$ & $\mathrm{H} \alpha$ & 415 & 125 & Henize (1956) \\
\hline $\mathrm{N}$ & $\mathrm{SMC}$ & $\mathrm{H} \alpha$ & 120 & 42 & Henize (1956) \\
\hline $\mathrm{DEM} \mathrm{L}^{\dagger}$ & $\mathrm{LMC}$ & $\mathrm{H} \alpha$ & 329 & 134 & Davies et al. (1976) \\
\hline DEM S & $\mathrm{SMC}$ & $\mathrm{H} \alpha$ & 167 & 46 & Davies et al. (1976) \\
\hline NGC (IC) & $\mathrm{LMC}$ & Optical & 182 & 67 & Sinnott (1980) \\
\hline NGC (IC) & $\mathrm{SMC}$ & Optical & 101 & 13 & Sinnott (1980) \\
\hline LH & LMC & Stellar Association & 122 & 61 & Lucke \& Hodge (1970) \\
\hline $\mathrm{Sa}$ & $\mathrm{LMC}$ & Planetary Nebulae & 128 & 5 & Sanduleak et al. $(1978,1984)$ \\
\hline PKS & $\mathrm{LMC}$ & Radio (Parkes) & 59 & 57 & Otrupcek \& Wright (1991) \\
\hline PKS & SMC & Radio (Parkes) & 24 & 23 & Otrupcek \& Wright (1991) \\
\hline $\mathrm{PMN}$ & $\mathrm{LMC}$ & $4.85 \mathrm{GHz}$ & 390 & 304 & Wright et al. (1994) \\
\hline PMN & $\mathrm{SMC}$ & $4.85 \mathrm{GHz}$ & 194 & 146 & Wright et al. (1994) \\
\hline $\mathrm{MC}^{\ddagger}$ & LMC & $5.00 \mathrm{GHz}$ & 95 & 80 & McGee et al. (1972a) \\
\hline S & SMC & $5.00 / 8.80 \mathrm{GHz}$ & 27 & 21 & McGee et al. (1976) \\
\hline $\mathrm{MC} 4$ & LMC & $408 \mathrm{MHz}$ & 227 & 202 & Clarke et al. (1976) \\
\hline MC4 & $\mathrm{SMC}$ & $408 \mathrm{MHz}$ & 63 & 56 & Clarke et al. (1976) \\
\hline MRC & SMC & $408 \mathrm{MHz}$ & 22 & 7 & Large et al. (1981) \\
\hline $\mathrm{CO}$ & LMC & $\mathrm{C}-\mathrm{O}^{13}(\mathrm{SEST})$ & 40 & 23 & Cohen et al. (1988) \\
\hline IJL & LMC & $\mathrm{C}-\mathrm{O}^{13}(\mathrm{SEST})$ & 92 & 53 & Israel et al. (1993) \\
\hline IJL & SMC & $\mathrm{C}-\mathrm{O}^{13}(\mathrm{SEST})$ & 42 & 14 & Israel et al. (1993) \\
\hline LI-LMC & $\mathrm{LMC}$ & IR (IRAS) & 1891 & 130 & Schwering \& Israel (1990) \\
\hline LI-SMC & SMC & IR (IRAS) & 249 & 38 & Schwering \& Israel (1990) \\
\hline LHG & $\mathrm{LMC}$ & X-ray (Einstein) & 97 & 32 & Long et al. (1981) \\
\hline $1 \mathrm{E}$ & $\mathrm{SMC}$ & X-ray (Einstein) & 28 & 9 & Seward \& Mitchell (1981) \\
\hline $\mathrm{W}$ & LMC & X-ray (Einstein) & 105 & 37 & Wang et al. (1991) \\
\hline IKT & SMC & X-ray (Einstein) & 25 & 9 & Inoue et al. (1983) \\
\hline WW & $\mathrm{SMC}$ & X-ray (Einstein) & 70 & 16 & Wang \& Wu (1992) \\
\hline BKGS & SMC & X-ray (Einstein) & 35 & 7 & Bruhweiler et al. (1987) \\
\hline RASS & $\mathrm{LMC}$ & X-ray (ROSAT) & 325 & 71 & Pietsch et al. (1997) \\
\hline RX J & $\mathrm{SMC}$ & X-ray (ROSAT) & 248 & 27 & Kahabka et al. (1997) \\
\hline
\end{tabular}

$\dagger$ DEM sources often contain more than one Henize source, e.g. DEM L10=N 79A,B,C,D,E. Therefore, DEM detected more sources in the MCs than Henize (1956).

${ }^{\ddagger}$ Three objects from MC catalogue out of the LMC field and two double.

1. background sources with $-1.8<\alpha<-0.6$

2. SNRs with a steep spectrum, $-0.8<\alpha<-0.2$ and

3. H II regions with a flat spectrum, $\alpha>-0.2$.

We use this criterion as a starting point to developing more precise criteria.

\subsubsection{Spectral-index distribution of the sources towards} the LMC

Figures 2a, 2b, and 2c show histograms of the source spectral-index distribution obtained for these sources using our determination of the spectral index. From the histograms it can be seen that the known $\mathrm{H}$ II regions have flat spectra $\left(\alpha_{\text {mean }}=-0.15\right.$ with standard deviation of 0.31$)$ and that SNRs and background sources have steeper spec- tra $\left(\alpha_{\text {mean }}=-0.43 \pm 0.19\right.$ and $\alpha_{\text {mean }}=-0.59 \pm 0.48$ respectively) (see Table 8).

Note that the SD of $\alpha$ for background sources is far larger than for the SNRs and H II regions. The reason for this is probably that background sources could be divided into two groups: one with steep spectra and the other with flat spectra. Variability of the background sources should be taken into account in understanding the large SD in $\alpha$. Source flux densities at different frequencies at different times for variable sources can give misleading spectralindex estimates. 
a)

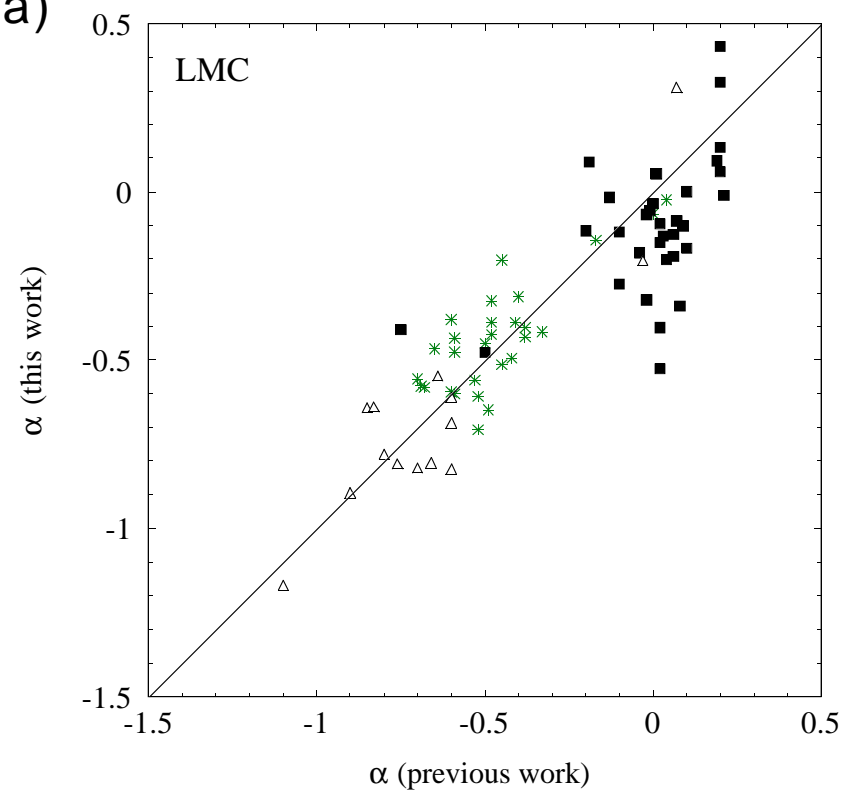

b)

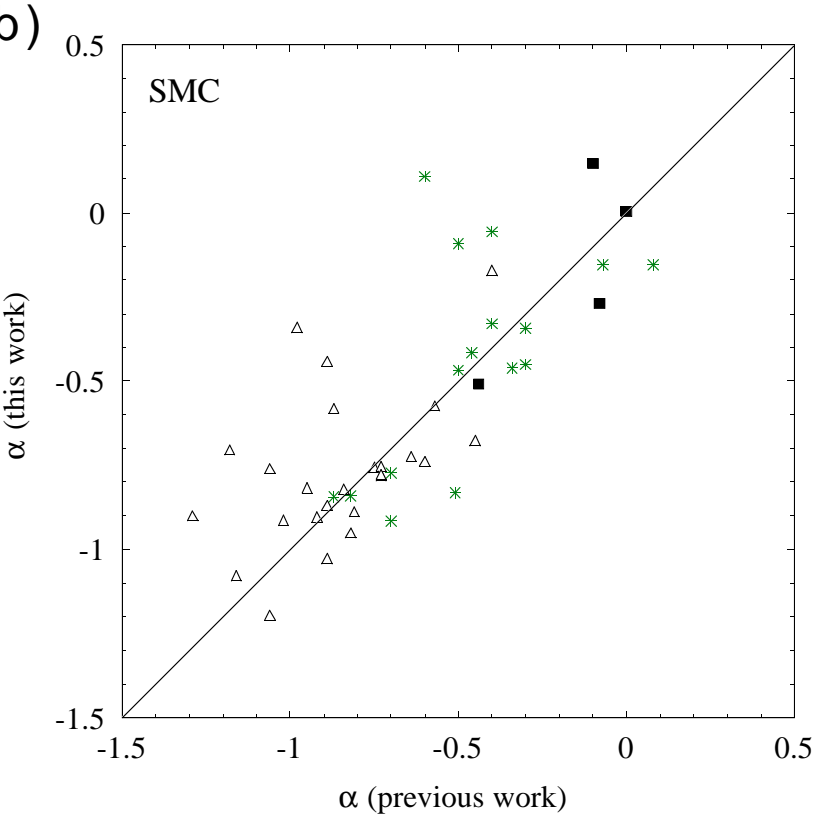

Fig. 1. Radio spectral index comparison for the LMC a) and the SMC b). Comparison of the radio spectra from previous work and this work. Asterisks represent SNRs; filled squares - H II regions and open triangles - background sources

Table 8. Spectral index distribution by source type in the LMC

\begin{tabular}{lccc}
\hline \hline $\begin{array}{l}\text { Source } \\
\text { Type }\end{array}$ & $\begin{array}{c}\text { No. of } \\
\text { Sources }\end{array}$ & $\begin{array}{c}\text { Mean } \\
\alpha\end{array}$ & $\begin{array}{c}\text { Standard } \\
\text { Deviation }\end{array}$ \\
\hline H II & 53 & -0.15 & 0.31 \\
SNRs & 32 & -0.43 & 0.19 \\
SNR candidates & 20 & -0.60 & 0.43 \\
Background & 68 & -0.59 & 0.48 \\
\hline
\end{tabular}

3.3.2. Spectral-index distribution of the sources towards the SMC

In the same way as for the LMC, in Figs. 3a, 3b and 3c are shown spectral index histograms of the same sources using the spectral index calculated in Sect. 3.1. (Table 6). From these histograms it can be seen that the known SMC H II regions have flat spectra $\left(\alpha_{\text {mean }}=0.03 \pm 0.09\right.$ with standard deviation of 0.34 ) and that SNRs and background sources have steeper spectra $\left(\alpha_{\text {mean }}=-0.22 \pm 0.07\right.$ and $\alpha_{\text {mean }}=-0.45 \pm 0.06$ respectively) (see Table 9 ). Again, Table 9 also shows that SD for background sources are far larger than for the SNRs and H II regions. The explanation for this is the same as for the LMC (see previous section).

3.3.3. Conclusions about the radio spectral index distribution

Sources cannot easily be classified by radio spectral index alone because of large overlaps in spectral indices between
Table 9. Spectral index distribution by source type in the SMC

\begin{tabular}{lcrc}
\hline \hline $\begin{array}{l}\text { Source } \\
\text { Type }\end{array}$ & $\begin{array}{c}\text { No. of } \\
\text { Sources }\end{array}$ & $\begin{array}{r}\text { Mean } \\
\alpha\end{array}$ & $\begin{array}{c}\text { Standard } \\
\text { Deviation }\end{array}$ \\
\hline H II & 14 & 0.03 & 0.34 \\
SNRs & 12 & -0.22 & 0.25 \\
Background & 63 & -0.45 & 0.48 \\
\hline
\end{tabular}

the various source types (also see Rosado et al. 1993b). In particular, SNRs and background sources (radio galaxies and quasars) have similar spectra resulting from synchrotron radiation. Furthermore, H II regions and SNRs are often associated, since a number of SNR in the MCs are embedded in or near to HII regions. It is thus not surprising that it is not possible to make an accurate classification on the basis of radio spectral index alone.

The distribution of spectral index for all of the 422 LMC and 164 SMC sources is given in Figs. 4a and $4 \mathrm{~b}$. The distribution is broad, covering the range $\alpha=-2.2$ to 1.1 for the LMC and $\alpha=-2.2$ to 1.5 for the SMC. However, most sources ( $86 \%$ in the LMC and $77 \%$ in the SMC) fall in the range -1.1 and 0.1 .

The mean spectral index for all sources towards the $\mathrm{LMC}$ is $-0.53 \pm 0.02(\mathrm{SD}=0.46)$ and towards the $\mathrm{SMC}$ is $-0.73 \pm 0.05(\mathrm{SD}=0.61)$. There is no significant difference in the mean spectral index after excluding of known background sources $\left(\alpha_{\text {mean }}=-0.51 \pm 0.02\right.$ with standard deviation 0.47 for the LMC and $\alpha_{\text {mean }}=-0.50 \pm 0.05$ with standard deviation 0.62 for the SMC). The mean spectral index for all sources towards the MCs agree well with 
a)

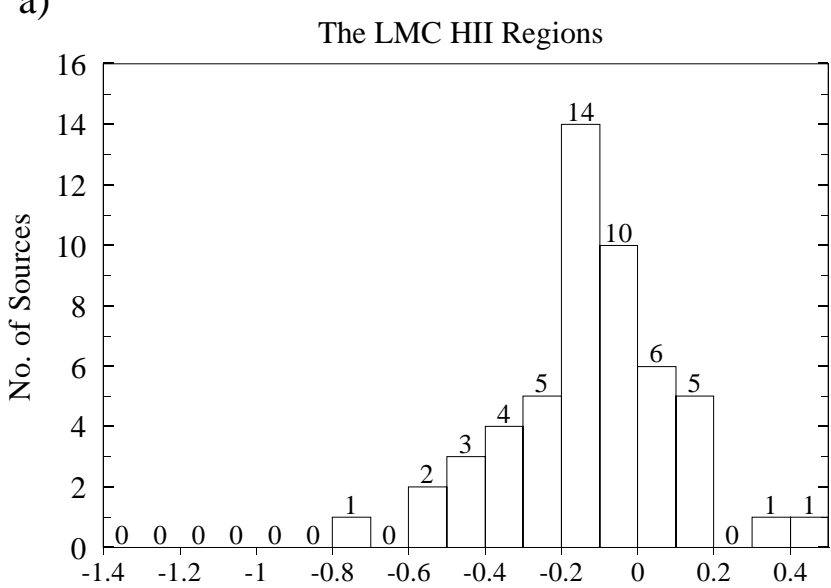

b)

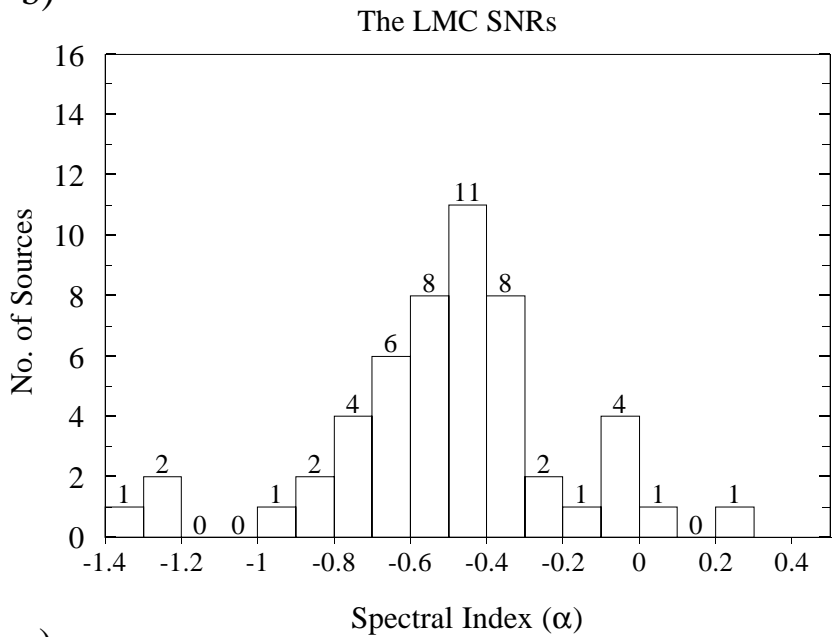

c)

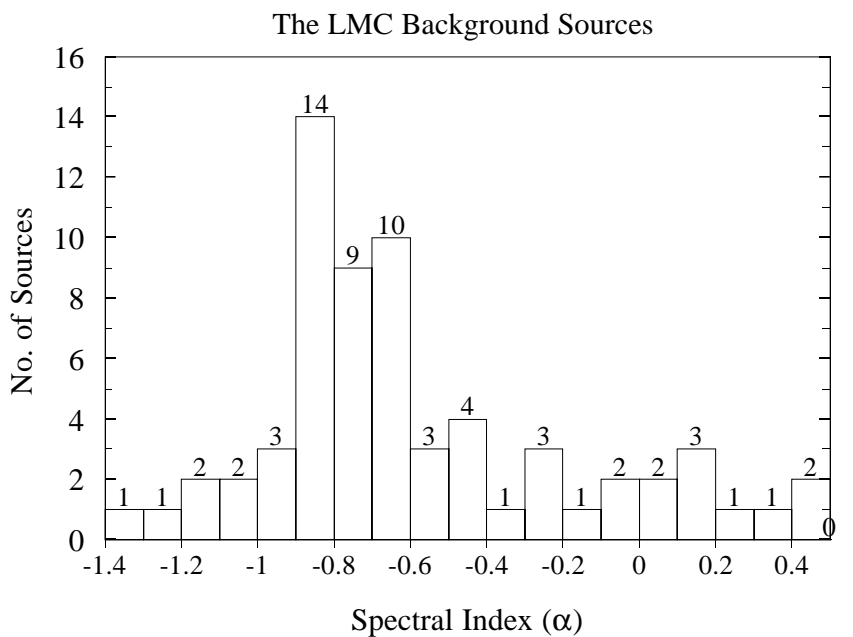

Fig. 2. Distributions of radio spectral index (this study) for the LMC sources which are classified in previous work (H II regions a), SNRs b) and background sources c)) a)

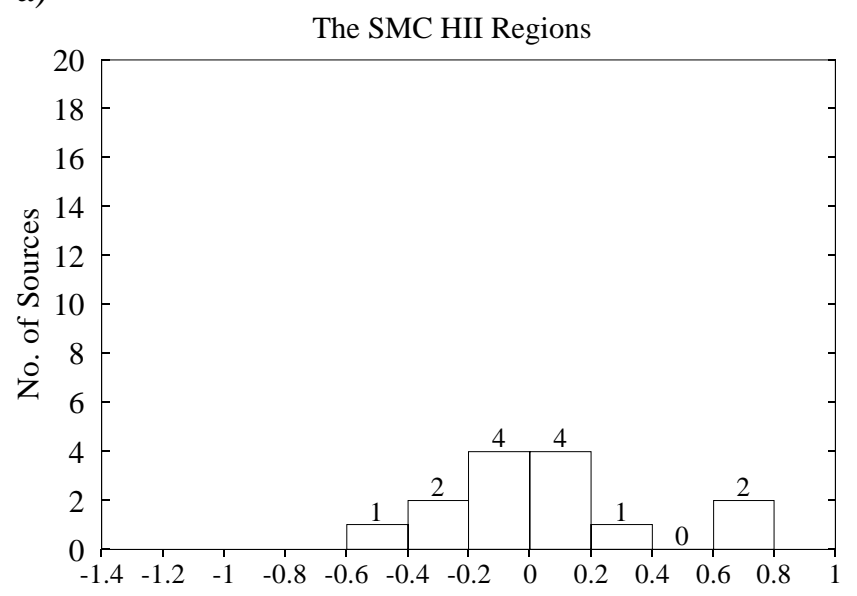

b)

Spectral Index $(\alpha)$

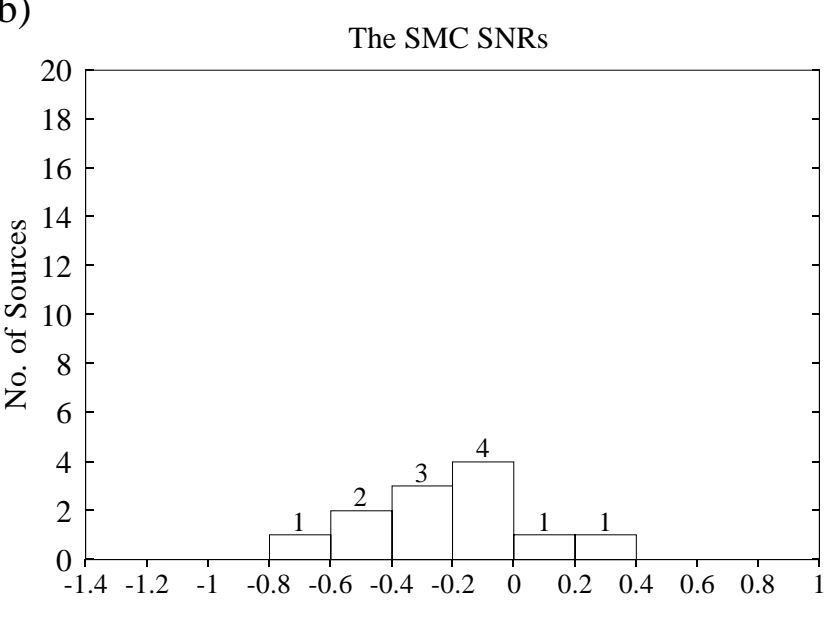

Spectral Index $(\alpha)$

c)

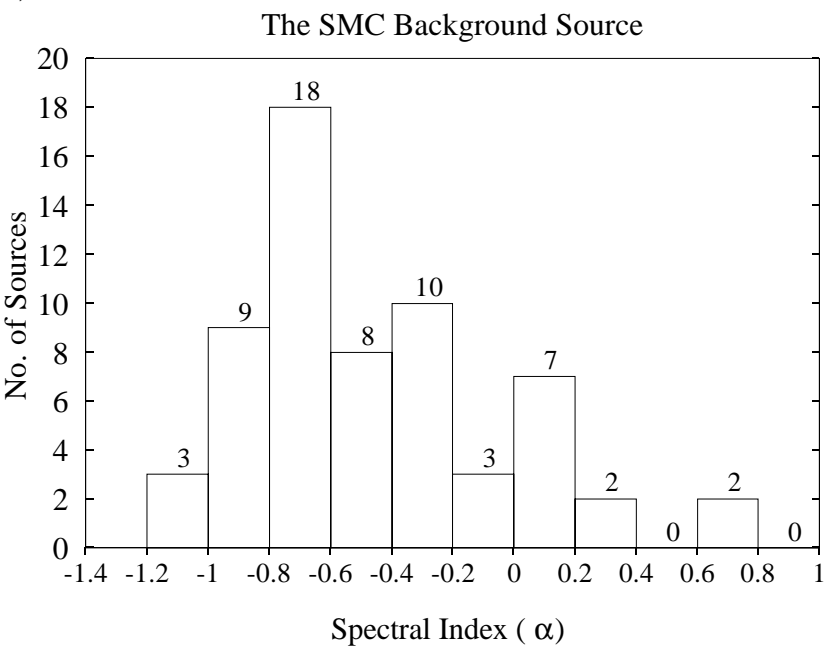

Fig. 3. Distributions of radio spectral index (this study) for the SMC sources which are classified in previous work (H II regions a), SNRs b) and background sources c)) 
a)

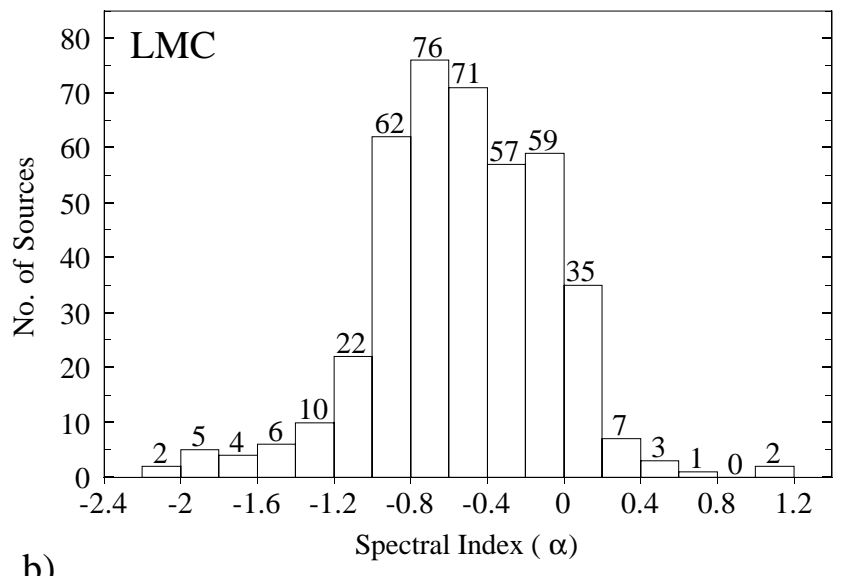

b)

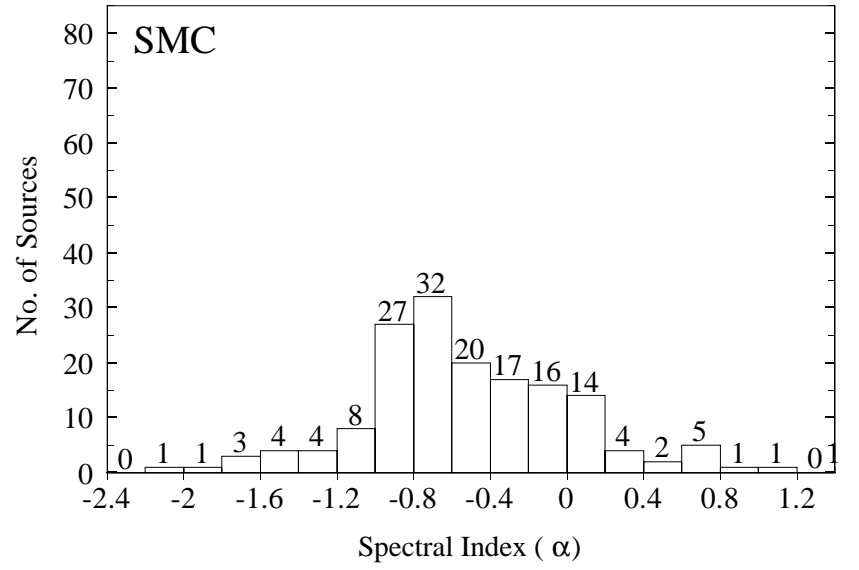

Fig. 4. Distributions of radio spectral index for the LMC a) and for the SMC b)

the overall spectral index including the diffuse component estimated by Haynes et al. (1991) and Klein et al. $(1989,1991)$. Their results are $-0.52 \pm 0.05$ for the LMC and $-0.78 \pm 0.11$ for the SMC.

Based on the classification scheme introduced by McGee \& Newton (1972), 57\% of sources towards the LMC and $61 \%$ sources towards the SMC show steep spectra $(\alpha \leq-0.45)$, and $27 \%$ of LMC and $21 \%$ of SMC sources have flat spectra $(\alpha \geq-0.20)$. The remaining sources, $16 \%$ for the LMC and $18 \%$ for the SMC, fall in the ambiguous region where $\alpha$ is between -0.44 and -0.21 .

\section{Classification and interpretation of the objects towards the Magellanic Clouds}

In previously published work, 192 radio sources towards the LMC and 93 towards the SMC have been classified as either H II regions, SNRs or background sources.

Of the 192 sources towards the LMC, 188 were detected in recent Parkes surveys (see Table 5). Of these 188 sources, 53 appear to be $\mathrm{H}$ II regions, 34 are confirmed
$\mathrm{SNRs}^{2}, 24$ are possible $\mathrm{SNRs}^{3}$ and 71 are listed as background sources ${ }^{4}$. Six sources are listed as ambiguous in Paper VI.

Four confirmed LMC SNRs listed in Mathewson et al. (1983a), Mills et al. (1984b), Tuohy et al. (1982) and Chu et al. (1997) could not be detected (B0505-679, B0509-675, B0543-689 and J0540-697) in any of our six surveys. We have discussed these four SNRs in Paper VI. Gurwell \& Hodge (1990), mainly using optical identifications, have reported a number of galaxies behind the LMC, but only a few of these have detectable radio emission.

The remaining 295 (483-188) sources listed in Table 5 provide new candidates for HII regions, SNRs and background galaxies or quasars. For 229 of these there is a fitted spectral index.

In the SMC, 102 sources were known and classified from previous radio investigations. Out of these, 92 were detected in the Parkes surveys (Table 6). Many of the SMC sources were discussed in Ye (1988). Of these 92 sources, 14 appear to be $\mathrm{H}$ II regions, 10 are confirmed SNRs, 2 are possible SNRs and 66 are listed as background sources.

Six confirmed SMC SNRs (SNR B0044-7324, B0045-7322, B0046-7335, B0056-7227, B0056-7223 and B0104-7221) and three SNR candidates (B0051-7259, B0056-7250 and B0057-7308) in the SMC could not be detected in any Parkes surveys because of their small angular size, weak radio emission and confusion. For the same reasons a number of background galaxies detected by Ye (1988) were missed.

The remaining 131 (224-92) unclassified sources (of which 73 have a fitted spectral index) towards the SMC are investigated to determine their nature.

\subsection{Selection criteria}

We have used the following criteria to classify the discrete radio sources listed in Tables 5 and 6 :

1. For Background source candidates -

background sources are either radio galaxies with generally steep radio spectra $(\alpha \leq-0.8)$ or quasars which have flat spectra $(\alpha \sim 0.0)$. Objects well removed from the stellar/nebular component of the MC are expected to be clear of the MCs objects and, by default, to be populated by background objects. Background objects are defined using following criteria.

- All radio sources outside the defined area of the LMC or the SMC are background sources. The area of the LMC is

${ }^{2}$ LMC B0454-6718 and LMC B0536-6920 (Honeycomb nebulae) were seen only in the $8.55-\mathrm{GHz}$ survey and hence no spectrum has been deduced.

3 LMC B0459-6612 was seen only in the 2.45-GHz and LMC B0540-6927 was seen only in the 8.55-GHz survey.

${ }^{4}$ LMC B0437-6420 was seen only in the 1.40-GHz survey. 
defined between $\mathrm{RA}(\mathrm{B} 1950)=04^{\mathrm{h}} 45^{\mathrm{m}} \quad$ to $\mathrm{RA}(\mathrm{B} 1950)=06^{\mathrm{h}} 00^{\mathrm{m}}$ and $\operatorname{Dec}(\mathrm{B} 1950)=-65^{\circ} 00^{\prime}$ to $\operatorname{Dec}(\mathrm{B} 1950)=-72^{\circ} 30^{\prime}$ : the area of the SMC as defined between $\mathrm{RA}(\mathrm{B} 1950)=00^{\mathrm{h}} 30^{\mathrm{m}}$ to $\mathrm{RA}(\mathrm{B} 1950)=01^{\mathrm{h}} 30^{\mathrm{m}}$ and $\operatorname{Dec}(\mathrm{B} 1950)=-71^{\circ} 40^{\prime}$ to $\operatorname{Dec}(\mathrm{B} 1950)=-74^{\circ} 00^{\prime}$.

- Radio sources with very steep spectra $(\alpha \leq-0.8)$ are more likely to be background objects. However, there is a wide range of radio spectral index for background sources.

- There is no $\mathrm{H} \alpha$ or IR detection (however some background sources can be detected in one of these frequencies but this is not likely) (Paper VIII).

2. For SNR and SNR candidates -

supernova remnants (SNRs) in the MCs have been the subject of numerous studies, both as individual SNRs and as a statistical sample. A series of criteria in several spectral ranges have been defined to identify SNRs in the MCs. Following Mathewson et al. (1983a and reference therein) and Rosado (1993b) the candidate must satisfy at least two of the following conditions. It should:

- be an extended soft X-ray source appearing either on Einstein surveys (Long et al. 1981; Wang et al. 1991) or the ROSAT all-sky survey (Pietsch \& Kahabka 1993; Pietsch et al. in preparation). A negative Hardness Ratio 2(HR2) indicates that the source is a SNR (Paper VI);

- be a non-thermal radio source $(\alpha=-0.4$ on average but there is a wide range and so spectral index is not itself conclusive) (Sect. 4);

- have optical emission with the following characteristics:

a) $[\mathrm{S} \mathrm{II}] /(\mathrm{H} \alpha+[\mathrm{N} \mathrm{II}]) \geq 0.4$

Possibly the most sensitive diagnostic for identifying SNRs is narrow-band optical CCD observations in the light of $\mathrm{H} \alpha$ and [S II]. The ratio of the $[\mathrm{S} \mathrm{II}] \lambda 6717,6713$ to $\mathrm{H} \alpha$ emission in nebulae provides a good discriminator between $\mathrm{H}$ II regions, which typically show [S II] $\mathrm{H} \alpha$ of $\sim 0.1$, and SNRs, which usually have ratios $>0.4$. This is because sulphur is ionized almost completely to the $\mathrm{S}^{++}$state in $\mathrm{H}$ II regions, while a wide range of temperatures and ionization states in the cooling regions behind SNR shocks gives rise to relatively strong [S II] emission;

b) have $\mathrm{H} \alpha$ with looped or filamentary structure and

c) an $\mathrm{H} \alpha$ flux density different from that expected for the radio counterpart in the case of thermal source (in the case of finding an H II region coincident with radio sources) (Ye 1988).

* have weak or no IR emission. SNRs are not likely to appear in IRAS surveys unless they are embedded in $\mathrm{H}$ II regions (Paper VIII).
These criteria are biased toward radio properties and further kinematical information is probably required. Furthermore, one must keep in mind that several wellknown SNRs in the MCs could not fulfil 2 of the 3 criteria given above because of the following:

- some radio SNRs could be missed in X-ray surveys if their X-ray emission is weak (or if it is attenuated by absorbing matter) (Paper VI);

- limitations in sensitivity of radio surveys. Some known MCs SNRs are weak radio emitters and near the sensitivity limit of the Parkes telescope, seen in only one radio frequency, and therefore have no radio spectral information. One example is DEM L298;

- confusion with the surrounding H II regions. Most of the LMC SNRs are embedded in H II regions.

3. For $\mathrm{H}$ II regions candidates -

$\mathrm{H}$ II regions are expected to be within the different nebular regions of the MCs. They are also expected:

* to be thermal radio sources (with $\alpha=-0.15$ on average) but because of wide range of spectral indices this criterion is not itself conclusive;

* Not to appear at X-ray frequencies - H II regions are not likely to be seen at X-ray frequencies (Paper VI);

* to have $\mathrm{H} \alpha$, UV and/or IR positive detections (Paper VIII).

\subsection{Background sources in the field of the $M C s$}

Because the MCs are transparent to radio continuum, background radio galaxies and quasars are seen throughout the MCs. It is very difficult to distinguish radio galaxies and quasars just on a basis of radio spectra and therefore, for this work, these sources will be treated together.

\subsubsection{Background sources in the field of the LMC}

Lists of background sources towards the LMC have been published in Gurwell \& Hodge (1990), Dickey et al. (1994) and Ye (1988). From their lists, 65 sources (marked in Table 5, Col. 17 as BG) are detected in our radio surveys as background, and an additional six as very probable background sources (marked in Table 5, Col. 17 as BG?). All of these 71 sources obey the criteria from Sect. 4.1. and will be treated as definite background sources.

Outside the defined area of the LMC, 97 sources (marked in Table 5 Col. 17 as bg) have been found. These sources are very strong candidates for background sources. Also, all of these sources obey the criteria established in Sect. 4.1. Another group of 34 sources (marked in Table 5, Col. 17 as bg?) satisfy some criteria of background sources and so are treated as background-source candidates.

In total, at least 202 sources towards the LMC are classified as background or background candidates, which 
is $42 \%$ of all sources found in the field of the LMC. Most of the 75 unclassified sources are likely to be background objects according to our source-density study (Sect. 4.2.3).

\subsubsection{Background sources in the field of the SMC}

The most complete list of background sources towards the SMC can be found in Ye (1988). From that list, 63 sources (marked in Table 6, Col. 14 as BG) are detected in our radio surveys as background and an additional three as very probable background sources (marked in Table 6, Col. 14 as BG?). All of these 66 sources obey the criteria from Sect. 4.2. and should be treated as definite background sources.

There are 108 sources outside the SMC area defined in Sect. 4.1. which have not been previously classified (marked in Table 6, Col. 14 as bg). These are very strong candidates for background sources as all of them satisfy criteria established in Sect. 4.1.

In total, at least 174 sources towards the SMC are classified as background or background candidates, which is $78 \%$ of all sources found in the field of the SMC. This number could increase slightly as the 12 unclassified sources are likely to be background according to the source-density study (Sect. 4.2.3).

\subsubsection{Statistics of the background sources}

The expected background integral source counts at our six survey frequencies were obtained by interpolating the polynomial fits ( $\log N-\log S$ study) of Wall (1994). These are the predicted number of background sources in the observed fields of the LMC and the SMC at each observing radio frequency (Tables 10 and 11).

Column 1 (Tables 10 and 11) lists the observing radio frequency and Col. 2 gives the $5 \sigma$ cut-off level in mJy. Column 3 lists the total number of sources found at each observing frequency and the area covered by each survey is given in Col. 4. The number of sources found in our surveys to be stronger then $5 \sigma$ at each frequency is given in Col. 5 and the total number of background sources stronger then $5 \sigma$ as given in Col. 6. The number of sources estimated from Wall (1994) for the given cut-off level (Col. 2) is given in Col. 7.

Comparing these results (Cols. 6 and 7), the number of background sources classified in this work (Sects. 4.2.1. and 4.2.2.) are very close to the expected range of the $\log N-\log S$ study. The agreement in numbers, at the $10 \%$ level, is as close as can be expected, given the sensitivity of integral counts to the flux-density calibration and flux cut-off. These findings strengthen our confidence in the selection criteria and source classification.

Figures 5 and 6 show the distribution of all classified background sources towards the LMC and the SMC. These source distributions are uniform, as expected.

\subsection{The Magellanic Clouds sources}

Radio sources intrinsic to the MCs are considered to be either SNRs or H II regions. SNRs are formed by the highvelocity and high-temperature material ejected when a star ends its life with a violent explosion. The notation "H II region" is usually reserved for relatively dense regions of the interstellar gas which are nearly completely ionized by the Lyman continuum radiation from massive and hot stars (Mezger 1972). H II regions in the MCs provide the opportunity to study the ionized gas component of the interstellar medium and star formation on a galactic scale, with a sensitivity and spatial resolution which are comparable to most of the Galactic surveys.

\subsubsection{Supernova remnants in the LMC}

Out of 62 SNRs (excluding the remnant of SN 1987A) and SNR candidates, 34 (marked in Table 5, Col. 17 as SNR) were confirmed to obey criteria from Sect. 4.1. and therefore will be treated as certain SNRs. Four well-known SNRs (see Sect. 4) could not be detected in any of our Parkes radio surveys. The other 24 sources (marked in Table 5, Col. 17 as SNR?), remain SNR candidates because of lack of confirmation. Thus only $12 \%$ (58 out of 483) are SNRs or SNR candidates. However, it is likely that more SNRs may exist which are confused with H II regions.

\subsubsection{H II regions in the LMC}

From previous studies, 53 radio sources (marked in Table 5, Col. 17 as $\mathrm{HII}$ ) are confirmed as HiI regions. They all obey the criteria established in Sect. 4.1. and therefore they are classified as certain $\mathrm{H}$ II regions. A further 41 previously unclassified sources (marked in Table 5, Col. 17 as h II) have characteristics typical for $\mathrm{H}$ II regions and hence they will be treated as very good candidates for H II regions. Another 20 sources (marked in Table 5, Col. 17 as hII?) obey at most two different criteria typical for $\mathrm{H}$ II regions and therefore they are suggested $\mathrm{H}$ II region candidates. Until further analysis one cannot be sure about the nature of these sources.

Of the 483 sources towards the LMC, 114 (24\%) are thus listed as $\mathrm{H}$ II regions. This number could increase as 34 sources (marked in Table 5, Col. 17 as ?) have some characteristics of MCs sources but one cannot be sure of their intrinsic nature.

\subsubsection{Supernova remnants in the $\mathrm{SMC}$}

From Ye's (1988) list of 19 SMC SNRs and SNR candidates, 10 (marked in Table 6, Col. 14 as SNR) were confirmed by the criteria from Sect. 4.1. and therefore they 
Table 10. $\log N-\log S$ estimates for the LMC

\begin{tabular}{|c|c|c|c|c|c|c|}
\hline $\begin{array}{c}(1) \\
\text { Frequency } \\
(\mathrm{GHz})\end{array}$ & $\begin{array}{c}(2) \\
5 \sigma \\
\mathrm{mJy}\end{array}$ & $\begin{array}{c}(3) \\
\text { Total No. } \\
\text { of Sources }\end{array}$ & $\begin{array}{c}(4) \\
\text { Area } \\
\left(\text { sq. }{ }^{\circ}\right)\end{array}$ & $\begin{array}{c}(5) \\
\text { No. of } \\
\text { Sources } \\
\geq 5 \sigma\end{array}$ & $\begin{array}{c}(6) \\
\text { No. of } \\
\text { Sources } \\
\text { (this work) }\end{array}$ & $\begin{array}{c}(7) \\
\text { No. of } \\
\text { Sources } \\
\text { (Wall 1994) }\end{array}$ \\
\hline 1.40 & 150 & 192 & 96.6 & 149 & $92 \pm 9$ & $87 \pm 9$ \\
\hline 2.30 & 150 & 119 & 99.8 & 110 & $49 \pm 7$ & $45 \pm 7$ \\
\hline 2.45 & 75 & 338 & 101.1 & 278 & $170 \pm 13$ & $152 \pm 12$ \\
\hline 4.75 & 40 & 373 & 77.0 & 334 & $161 \pm 13$ & $177 \pm 13$ \\
\hline 4.85 & 25 & 332 & 56.7 & 329 & $182 \pm 14$ & $170 \pm 13$ \\
\hline 8.55 & 45 & 212 & 39.9 & 194 & $45 \pm 7$ & $40 \pm 6$ \\
\hline
\end{tabular}

Table 11. $\log N-\log S$ estimates for the SMC

\begin{tabular}{ccccccc}
\hline \hline $\begin{array}{c}(1) \\
\begin{array}{c}\text { Frequency } \\
(\mathrm{GHz})\end{array}\end{array}$ & $\begin{array}{c}(2) \\
\text { mJy }\end{array}$ & $\begin{array}{c}(3) \\
\text { Total No. } \\
\text { of Sources }\end{array}$ & $\begin{array}{c}(4) \\
\text { (sq. }\end{array}{ }^{\circ}$ ) & $\begin{array}{c}(5) \\
\text { No. of } \\
\text { Sources } \\
\geq 5 \sigma\end{array}$ & $\begin{array}{c}(6) \\
\text { No. of } \\
\text { Sources } \\
\text { (this work) }\end{array}$ & $\begin{array}{c}(7) \\
\text { No. of } \\
\text { Sources } \\
\text { (Wall 1994) }\end{array}$ \\
\hline 1.40 & 75 & 85 & 40.91 & 83 & $76 \pm 9$ & $90 \pm 9$ \\
2.45 & 50 & 107 & 37.01 & 103 & $87 \pm 9$ & $70 \pm 8$ \\
4.75 & 30 & 99 & 26.85 & 95 & $66 \pm 8$ & $62 \pm 8$ \\
4.85 & 25 & 187 & 40.91 & 179 & $150 \pm 12$ & $123 \pm 11$ \\
8.55 & 50 & 41 & 8.77 & 39 & $15 \pm 4$ & $11 \pm 3$ \\
\hline
\end{tabular}

are certain SNRs. Six well-known SNRs and three SNR candidates (see Sect. 4) could not be detected in any of our Parkes radio surveys due to the flux density limits. The remaining two SNR candidates (marked in Table 6, Col. 14 as SNR?) are still under study and, at present, they will remain as SNR candidates.

Only $6 \%$ (12 out of 224 ) of sources towards the SMC shows characteristics of SNRs. However, as for the LMC, it is likely that this number of SNRs could be higher because of their confusion with $\mathrm{H}$ II regions.

\subsubsection{H II regions in the SMC}

From previous studies, 14 radio sources listed in Paper V (and also marked in Table 6, Col. 14 as H II) are confirmed to be $\mathrm{H}$ II regions. They obey all criteria for $\mathrm{H}$ II regions (Sect. 4.1.) and therefore they are classified as certain HII regions. Another ten previously unclassified sources (marked in Table 6, Col. 14 as h II) have all characteristics typical for $\mathrm{H}$ II regions and hence they will be treated as very good candidates for $\mathrm{H}$ II regions.

Of the 224 sources towards the SMC, only 24 (11\%) are listed as $\mathrm{H}$ II regions or $\mathrm{H}$ II region candidates. Two other sources (marked in Table 6 Col. 14 as ?) also have some characteristics of $\mathrm{H}$ II regions but their status also remains unclear.

\section{Luminosity functions of SNRs and $H$ II regions in the MCs}

The luminosity of SNRs and H II regions is important in understanding the physical nature of the sources. For sources of the same distance, such as those in the MCs, the luminosity is directly related to the observed flux without the extra complication of uncertain distance estimates. Therefore, the luminosity function can be obtained much more directly than for our Galaxy.

The luminosity of each radio source is given in $\mathrm{W} \mathrm{Hz}^{-1}$ and is defined by the relation:

$L=4 \pi D^{2} S$

where $D$ is distance to the Clouds $(50 \mathrm{kpc}$ to the LMC and $60 \mathrm{kpc}$ to the SMC; Westerlund 1993) and $S$ is the flux density at the given radio frequency (1 $\left.\mathrm{Jy}=10^{-26} \mathrm{~W} \mathrm{~m}^{-2} \mathrm{~Hz}^{-1}\right)$.

Source flux densities at $4.75 \mathrm{GHz}$ (for the LMC) and $4.85 \mathrm{GHz}$ (for the SMC) were used to estimate the luminosity of each source. The completeness level at the $4.75 \mathrm{GHz}$ LMC surveys is $40 \mathrm{mJy}$ (the $5 \sigma$ level) (for more details see Table 9; Col. 2) and for the 4.85-GHz SMC survey is $25 \mathrm{mJy}$ (the $5 \sigma$ level) (for more details see Table 10; Col. 2). These correspond to completeness levels in luminosity of $1.210^{16}$ and $1.110^{16} \mathrm{~W} \mathrm{~Hz}^{-1}$ for the LMC and the SMC respectively. 


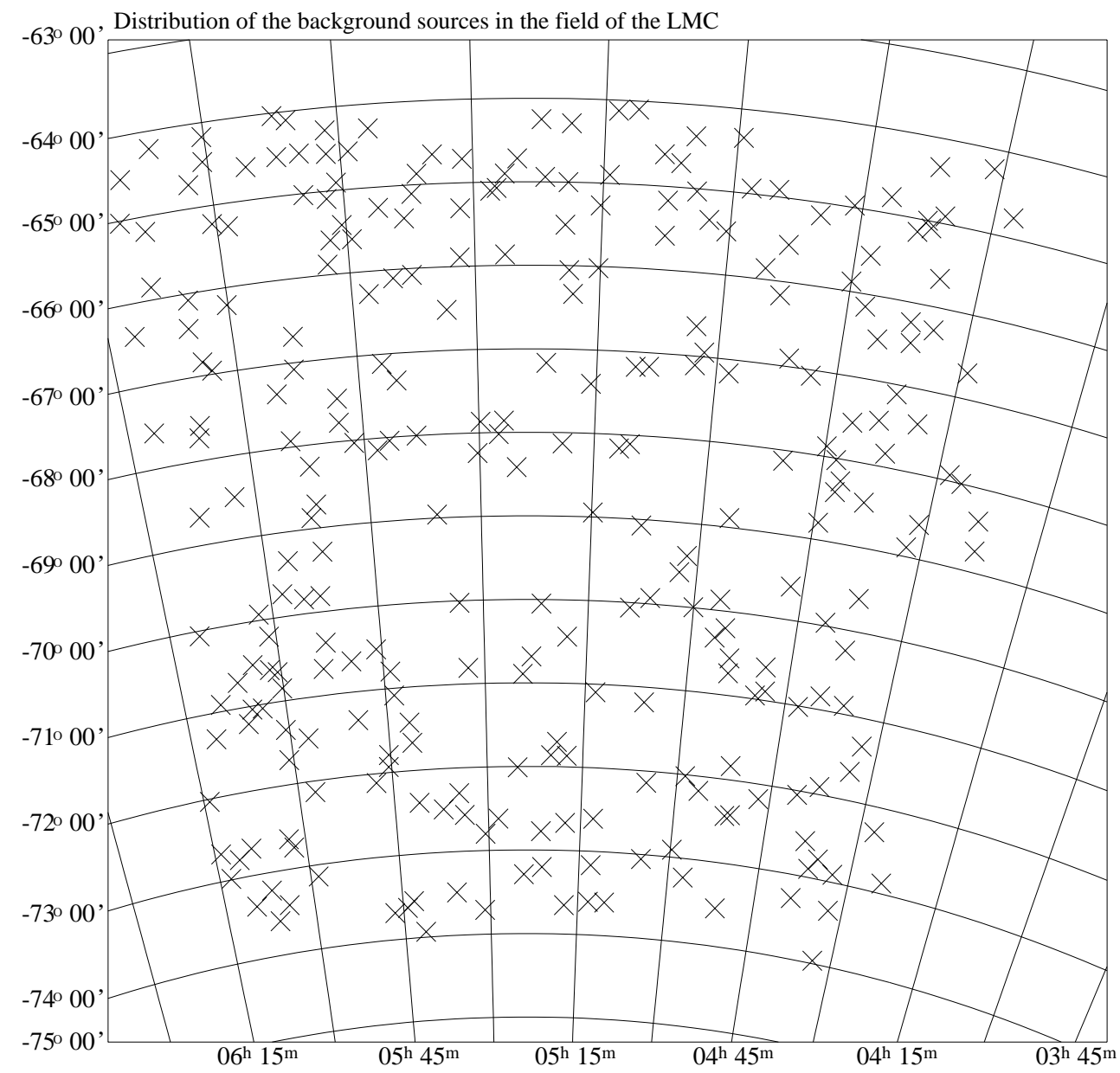

Fig. 5. Distribution of background sources towards the LMC

\subsection{The luminosity function of SNRs in the $L M C$}

Figures $7 \mathrm{a}$ and $7 \mathrm{~b}$ show the histogram of luminosities for SNRs and SNR candidates in the LMC. We have flux densities at $4.75 \mathrm{GHz}$ for 53 of these. The SNRs without flux density at this frequency, are plotted in the lowest bin in Fig. 7 a since they are below the $5 \sigma$ level.

In Fig. 7a the highest luminosity sources are above the plotted range. To show the full range of luminosities, the luminosity distribution is plotted also in Fig. 7b on a logarithmic scale with equal bins in the log domain (rather than linear bins as in Fig. 7a).

As expected, there are more sources with low luminosity than with high luminosity. To quantify the distribution a power law has been fitted and this gives a power-law index of -1.2 (i.e. $N(L) \propto L^{-1.2} \mathrm{~d} L$ ). The theory for the evolution of SNR diameters and luminosity is not well understood. However, the expected evolution of SNR diameter and surface brightness is generally described by power laws as a function of time (Lozinskaya 1992). Therefore, the expected evolution of luminosity is a power law with time, and the luminosity function is a power law assuming that SN explosions occur at a constant rate in the LMC.

We do not have either sensitivity or spatial resolution to determine the phase of evolution (Sedov or linear expansion) of any SNRs in the LMC. Confusion (in most cases) with surrounding $\mathrm{H}$ II regions further worsens the situation. Therefore, the luminosity function power-law index estimated here can be understood as an overall powerlaw index for SNRs in the LMC, combining SNRs in all different phases of evolution.

\subsection{The luminosity function of $\mathrm{H} \mathrm{II}$ regions in the LMC}

The luminosity function of the LMC H II regions was calculated in a similar way to the LMC SNRs. The $148 \mathrm{H} \mathrm{II}$ regions (and $\mathrm{H}$ II region candidates) in the LMC are plotted in the histogram (Fig. 8a) where we have 124 objects with $4.75-\mathrm{GHz}$ flux densities, and the rest are plotted (as weak sources below the $5 \sigma$ level) in the lowest bin.

Figure $8 \mathrm{a}$ does not plot the highest luminosity HII regions in the LMC and therefore Fig. 8b shows luminosity 


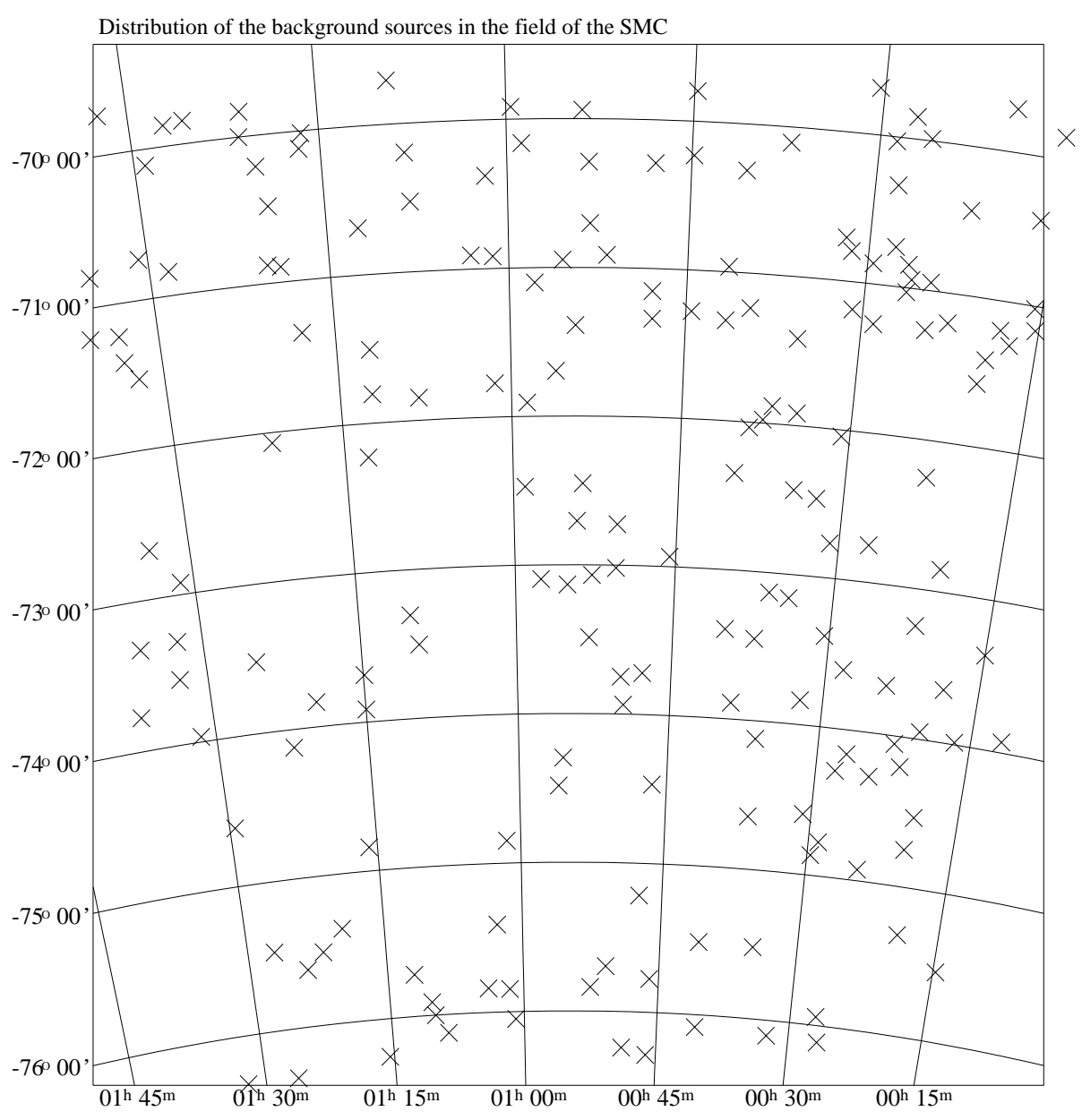

Fig. 6. Distribution of background sources towards the SMC

on a logarithmic scale with log bins. This plot does not include $\mathrm{H}$ II regions which are not detected in the 4.75$\mathrm{GHz}$ survey. Note that the most luminous H II region is 30 Doradus which is two orders of magnitude stronger than for "typical" H II regions.

The distribution in luminosity has been fitted with an exponential curve $\left(N(L) \propto \exp \left(-L / L_{0}\right) \mathrm{d} L\right)$ with characteristic luminosity scale $L_{0}=4.210^{16} \mathrm{~W} \mathrm{~Hz}^{-1}$. Ye (1988) fitted the diameter distributions for $\mathrm{HII}$ regions as an exponential function and using a similar method he has also fitted the luminosity distribution with an exponential function for the MCs and other galaxies.

\subsection{The luminosity function of SNRs in the $S M C$}

Because of the small number of the SMC SNRs (12), the luminosity function is rather sparse. However, similar histograms to those from Sect. 5.1. are plotted in Figs. 9a and $9 \mathrm{~b}$. Despite the small number of sources, the luminosity distribution looks similar to that of the LMC SNRs.
The luminosity function for SMC SNRs is shown in Fig. 9a. The luminosity distribution is plotted also in Fig. 9b on a logarithmic scale with equal bins in the log domain (rather then linear bins as in Fig. 9a). Only the SNRs detected at the $4.85-\mathrm{GHz}$ survey are plotted in Fig. 9b.

\subsection{The luminosity function of $\mathrm{H} \mathrm{II}$ regions in the SMC}

The number of SMC H II regions is also small (26); however, the luminosity functions are plotted in Figs. 10a and 10b. The most luminous SMC H II region is not nearly as bright as 30 Doradus in the LMC.

In Fig. 10a, the histogram is plotted of the SMC H II region luminosities and Fig. 10b shows the luminosity function on a logarithmic scale with log bins. This plot does not include $\mathrm{H}$ II regions which are not detected at the 4.85GHz survey. 
a)

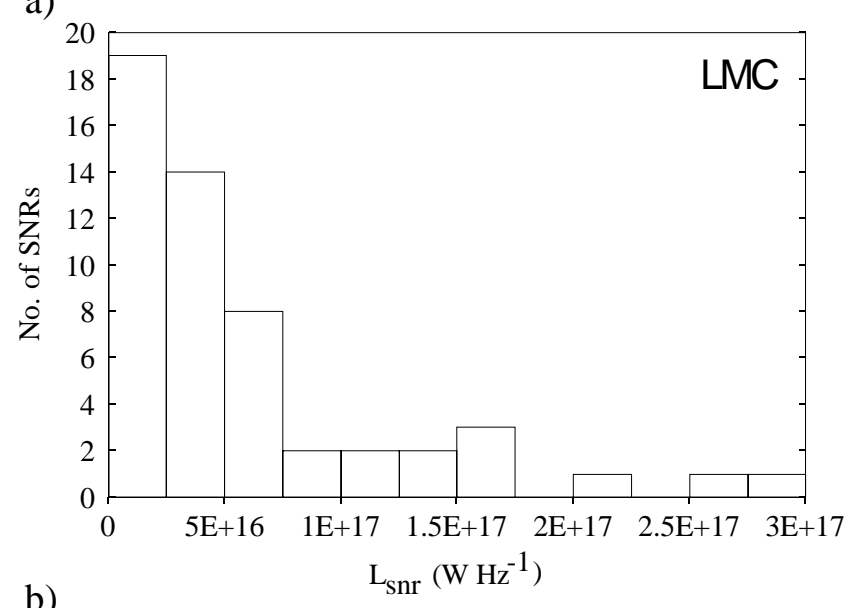

b)

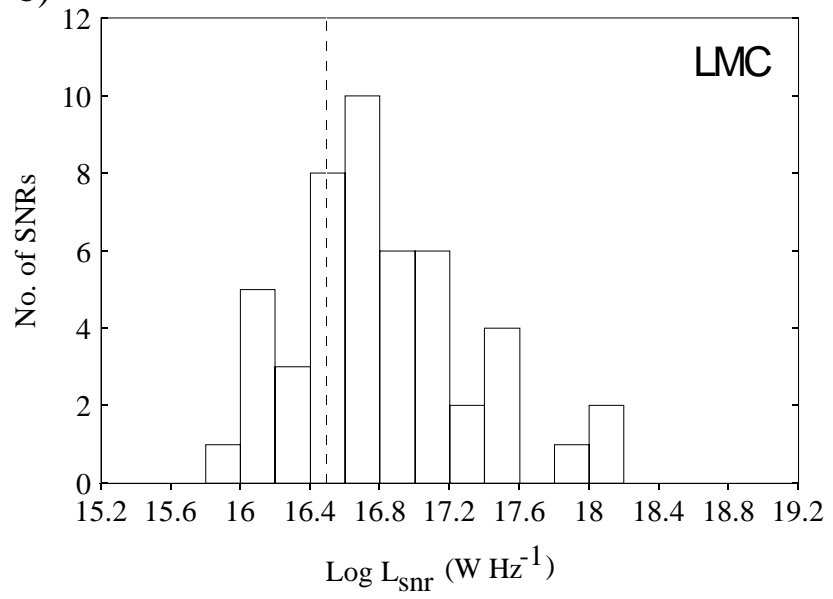

Fig. 7. The luminosity function of SNRs in the LMC. In Fig. 7a the luminosity distribution is plotted on a linear scale and in Fig. $7 \mathrm{~b}$ it is plotted on a logarithmic scale with logarithmic bins. The vertical dashed line represent the approximate completeness level assuming the $S_{4.75}$ flux densities to be complete down to $\sim 0.1 \mathrm{Jy}$

\section{Source distribution throughout the MCs}

Fujimoto \& Kumai (1990) have proposed a model of the collision between the LMC and SMC about 0.2 Gyr ago (see also Murai \& Fujimoto 1980; Mathewson et al. 1987). In this model the SMC should have penetrated through the LMC in the area around RA (B1950) $=05^{\mathrm{h}} 20^{\mathrm{m}}$ and Dec $(B 1950)=-70^{\circ}$ where we now find the lowest intensity of the radio emission. This is also the area of the steepest observed non-thermal spectral index (Meinert 1992). The proposal is that the SMC swept away the thermal gas along its track thus forming the bridge between the Clouds and initiating the Magellanic Stream. Fujimoto \& Kumai (1990) concluded that during this collision the gas in the LMC was forced to rotate around the intruder galaxy, probably in a plane different from that of the LMC. This may have resulted in the asymmetric distribution of both atomic and molecular gas which is found mainly south of a)

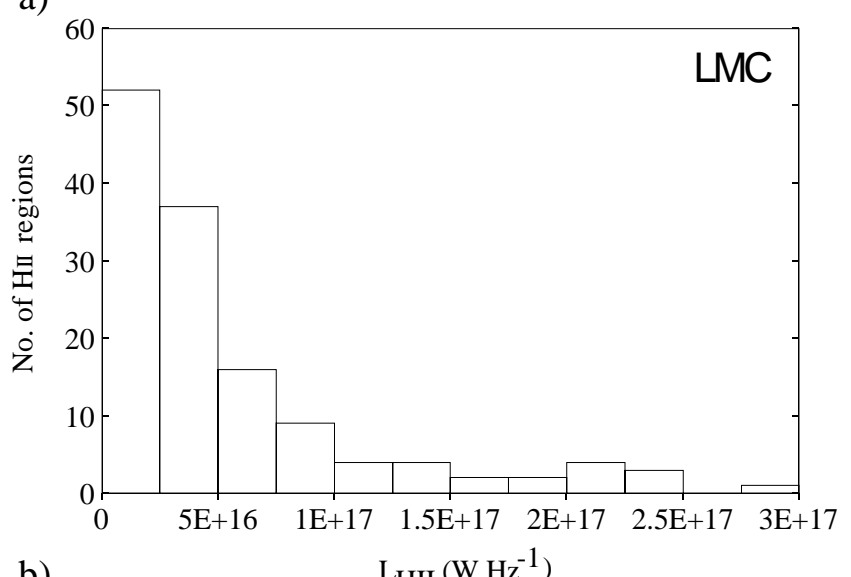

b)

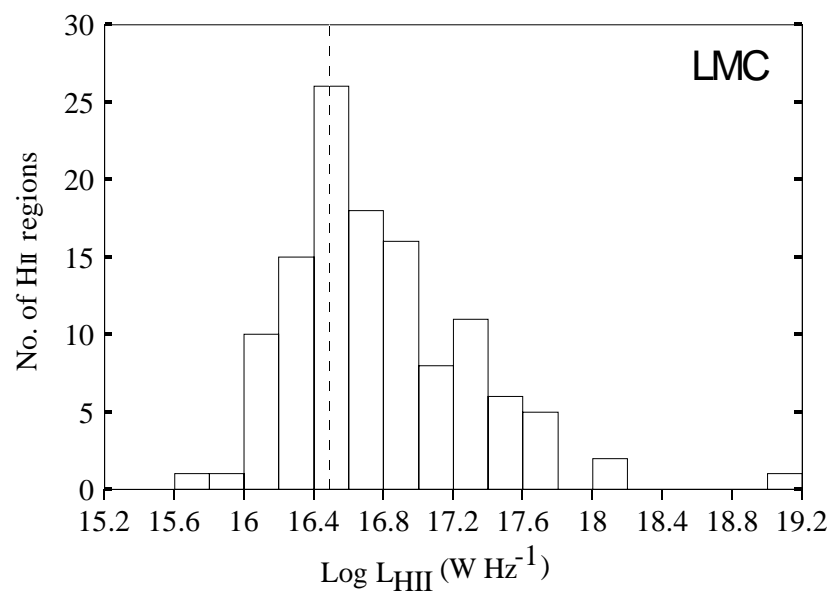

Fig. 8. The luminosity function of $\mathrm{H}$ II regions in the LMC. In Fig. 8a the luminosity distribution is plotted on a linear scale and in Fig. 8b it is plotted on a logarithmic scale with logarithmic bins. The vertical dashed line represent the approximate completeness level assuming the $S_{4.75}$ flux densities to be complete down to $\sim 0.1 \mathrm{Jy}$

the 30 Doradus complex. Another remnant of this collision may be the $L$-component, which was recently detected in H I gas (Luks \& Rohlfs 1992). Probably this model can also account for self-propagating star formation in this area.

Consider the following model. If the rotation of the gas in the LMC were disrupted in the region of the collision, perhaps by shock processes mentioned above, the density would increase and eventually a Jeans instability would occur. This mass assembly could also stop the gas coming in later along the same track and, because the primary complex has already started forming stars, it gave rise to pressure against the infall. Eventually this may induce another new complex of star formation in the direction of the infalling gas stream. If this model is correct 30 Doradus may be the latest, already evolved, of those star- forming complexes - probably preceded by the Shapley III complex - and it may have triggered star formation in the 30 Doradus complex. 

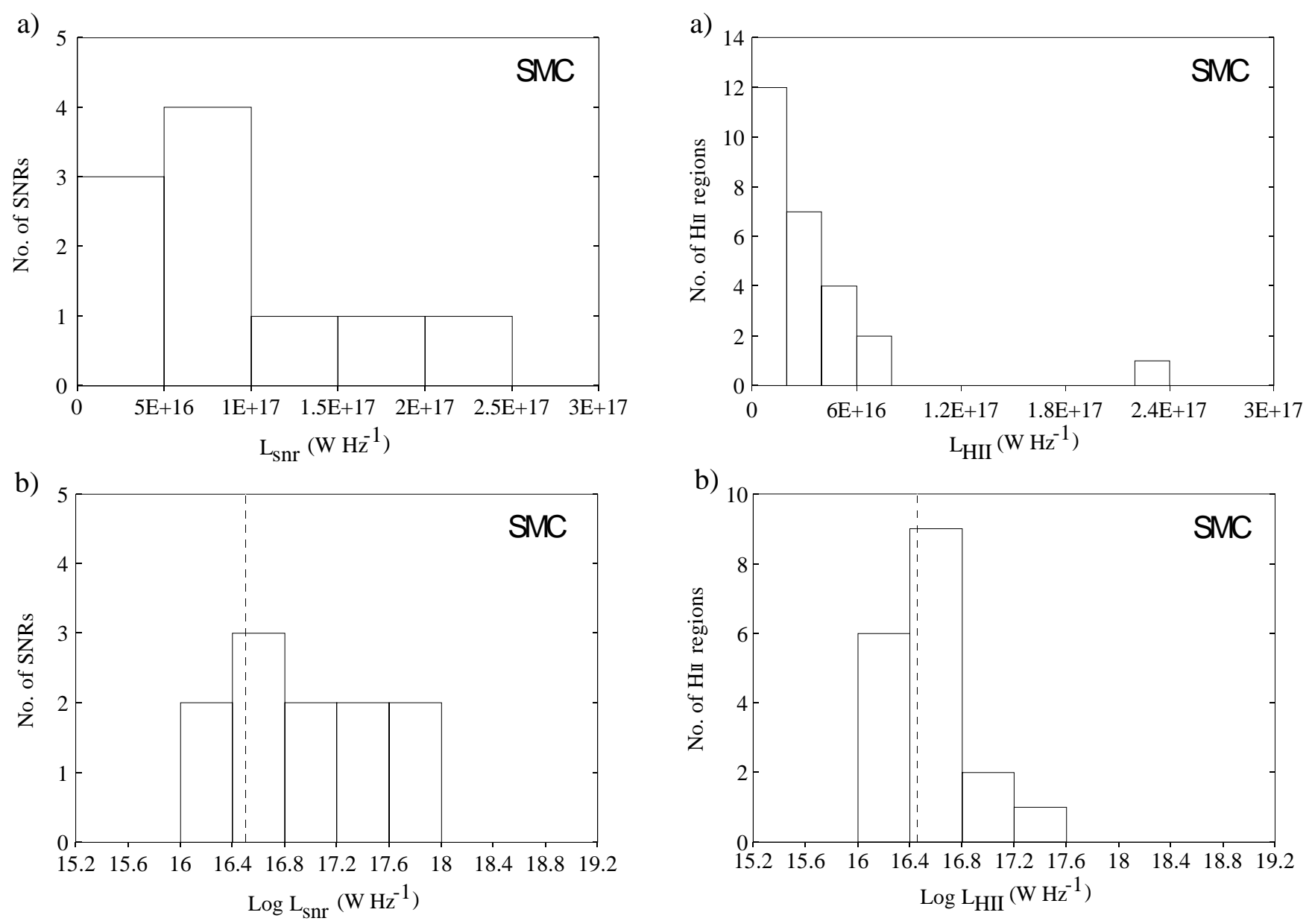

Fig. 9. The luminosity function of SNRs in the SMC. In Fig. 9a the luminosity distribution is plotted on a linear scale and in Fig. 9b it is plotted on a logarithmic scale with logarithmic bins. The vertical dashed line represent the approximate completeness level assuming the $S_{4.75}$ flux densities to be complete down to $\sim 0.1 \mathrm{Jy}$

Fig. 10. The luminosity function of HII regions in the SMC. In Fig. 10a the luminosity distribution is plotted on a linear scale and in Fig. 10b it is plotted on a logarithmic scale with logarithmic bins. The vertical dashed line represent the approximate completeness level assuming the $S_{4.75}$ flux densities to be complete down to $\sim 0.1 \mathrm{Jy}$

\subsection{Source 2-D distribution throughout the LMC}

Taking into account the ram pressure of the galactic halo gas onto the LMC interstellar medium (Meurer et al. 1985), it may be possible to give an alternative explanation for this star-forming complex. Ram pressure may well have compressed the gas at the border, and since the LMC is rotating in a clockwise sense the 30 Doradus complex would have reached this shock front earlier than N 159. In favour of the previous model it should be noted that the large gas cloud lies almost perpendicular to the bow shock, and so one would expect star formation all along its eastern ridge, not only at its northern end.

In order to understand the overall structure of the MCs, we plot the positions of sources intrinsic to both Clouds.

The source distribution throughout the LMC is shown in Fig. 11. All SNRs and SNR candidates (62) are plotted together with $148 \mathrm{H}$ II regions (and $\mathrm{H}$ II region candidates).

Smith et al. (1987) first gave the 2-D distribution of the LMC discrete sources but for a limited number of sources $(\sim 20)$. Here, with a significantly improved number of LMC sources (209), we found that the LMC sources follow patterns initially indicated by Shapley (1956) and Martin et al. (1976). These patterns are possible spiral arms, which were introduced by Feitzinger et al. (1987 and reference therein) and Schmidt-Kaler (1993 and reference therein). From Fig. 11 it can be seen that some regions throughout the LMC do not have strong or obvious sources. Three such a regions in the LMC were observed here: 


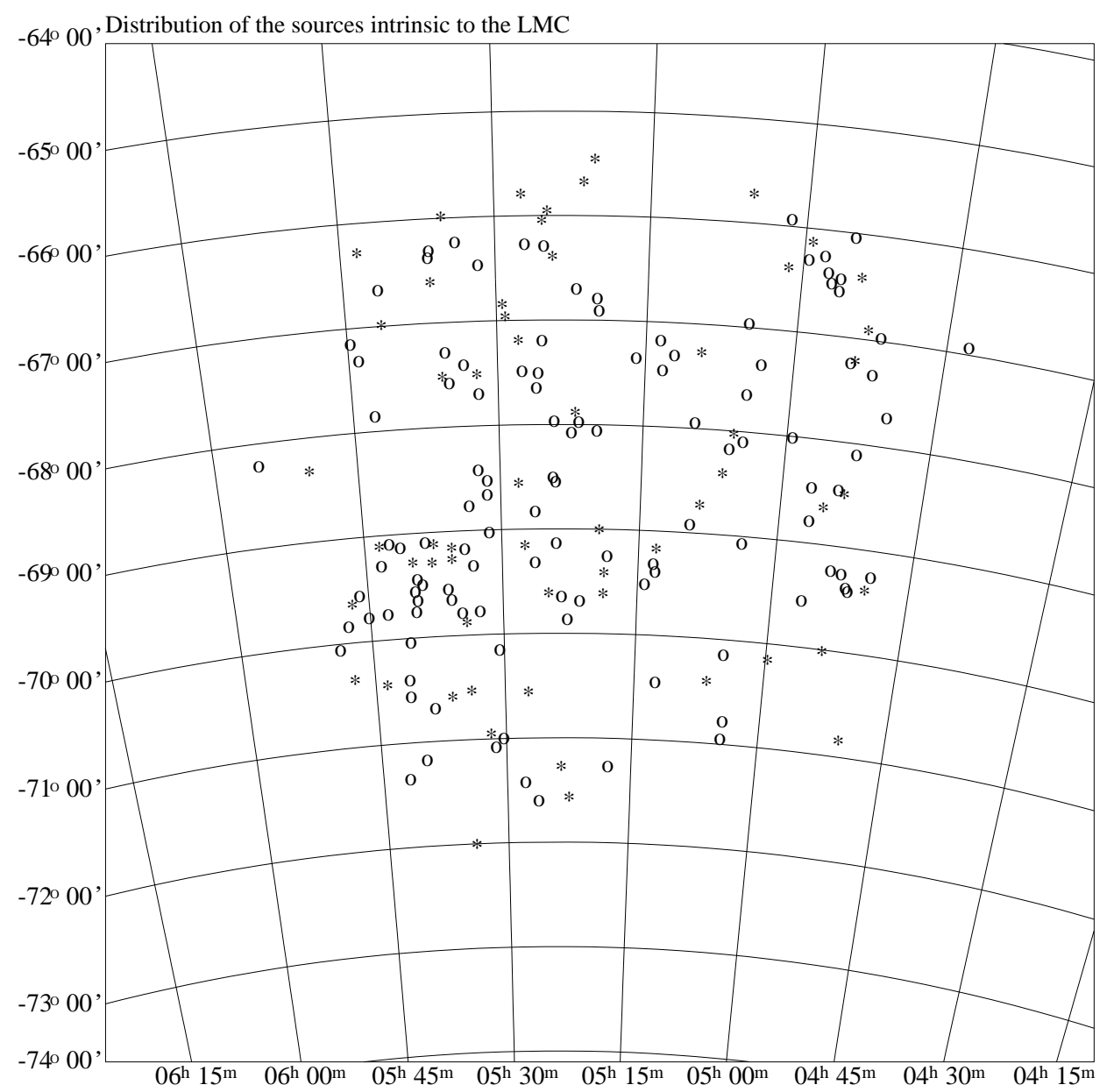

Fig. 11. The distribution of the radio sources intrinsic to the LMC. Asterisks represent SNRs and SNR candidates and open circles represent $\mathrm{H}$ II regions and $\mathrm{H}$ II region candidates

1. the region north from 30 Doradus region with centre at $\sim \mathrm{RA}(\mathrm{B} 1950)=05^{\mathrm{h}} 38^{\mathrm{m}}$ and $\operatorname{Dec}(\mathrm{B} 1950)=-68^{\circ} 20^{\prime}$. This region extends approximately for a diameter of some $50^{\prime}$ from this centre;

2. the region south of the optical bar, between the LMC Complexes A2', A2, A1 and the LMC Complexes B3, $\mathrm{B} 2^{\prime}, \mathrm{B} 21$ and

3. the region between Shapley Constellation III, Shapley Constellation IV and the LMC Complex B1.

Generally, the distribution of the intrinsic sources throughout the LMC indicates some kind of spiral structure with a possible centre in the region around or close to 30 Doradus. According to Feitzinger et al. (1987), this large-scale spiral pattern consists of as many as six or seven arm-like features. On the other hand it is difficult to explain why such a young complex as 30 Doradus is a centre of evolved spiral structure. Also, it is very difficult to understand why the radio continuum picture of the LMC is so different from the optical, where the optical bar is so dominant. The optical bar of the LMC is not directly related to the spiral pattern; it probably reflects an earlier period of evolution of the galaxy. These questions could be answered in the new high-resolution $\mathrm{H}$ I survey of the LMC (L. Staveley-Smith private communication 1997). Also, the sharp eastern end on all continuum images of the LMC is noted. Similar tendencies can be found in the ROSAT X-ray surveys (Pietsch et al. in preparation). We believe that this is caused by the ram pressure of the LMC motion toward us.

\subsection{Source 2-D distribution throughout the $S M C$}

The SMC is much less rich than the LMC in intrinsic discrete sources. Only $37 \mathrm{SMC}$ sources can be seen from our surveys and they are plotted in Fig. 12. Two regions (main-body of the SMC and eastern wing of the SMC) are dominant. However, we cannot confirm the depth of these regions and the distribution of sources along the line of sight with our observations. 


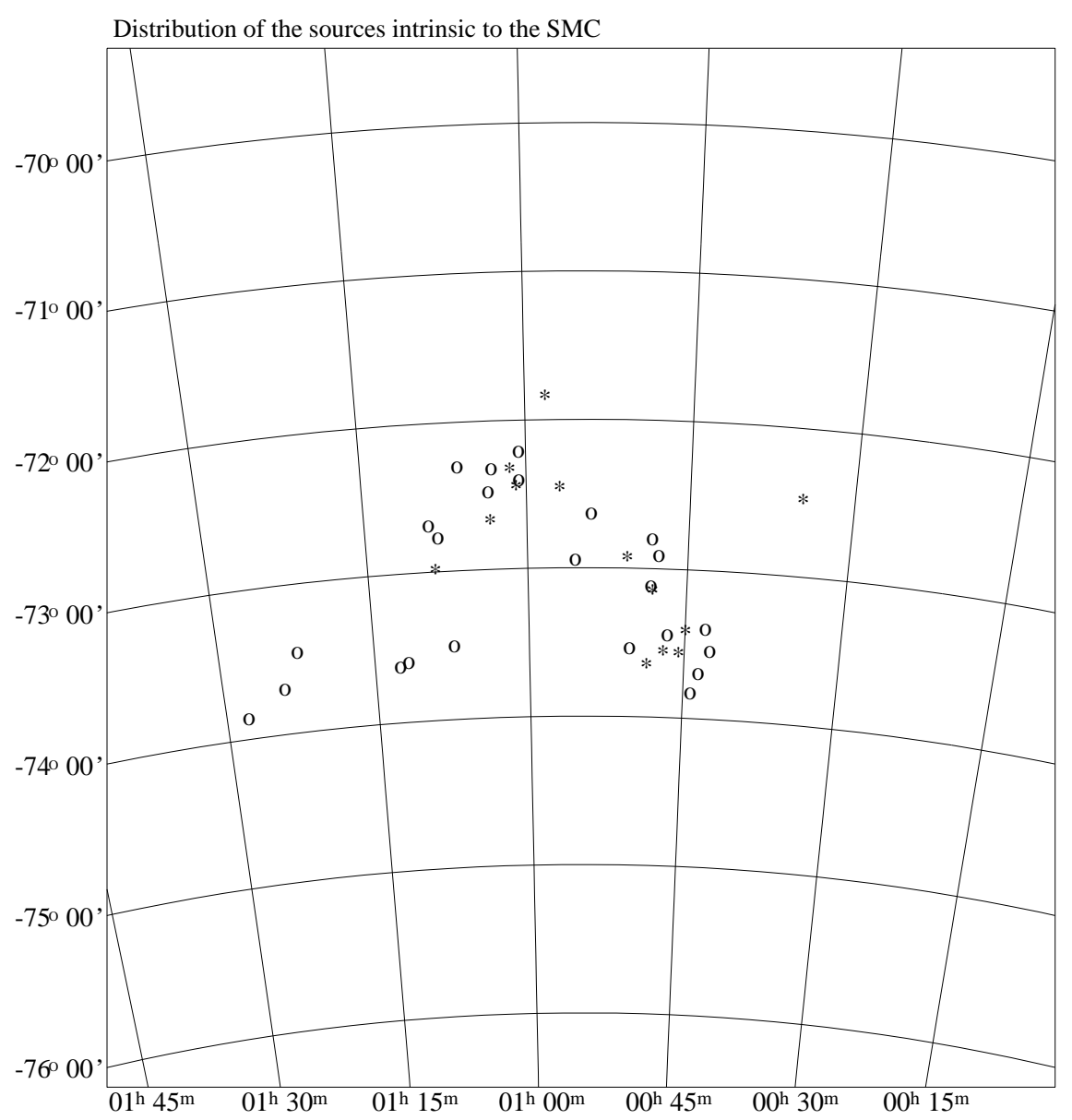

Fig. 12. The distribution of the radio sources intrinsic to the SMC. Asterisks represent SNRs and SNR candidates and open circles represent $\mathrm{H}$ II regions and $\mathrm{H}$ II region candidates

Here, we note the known SMC X-ray binary system (1E 0035.4-7230; Kahabka \& Pietsch 1996) which is some $\sim 60^{\prime}$ on the far west side from the rest of the SMC. Why such a source is so distant from the rest of the galaxy at present remains a mystery.

\section{Concluding remarks}

This paper presents new catalogues of source radio spectral index in the MCs. There is a total of 483 radio sources towards the LMC, 143 of which are classified for the first time. Also new catalogues of source radio spectral index in the SMC are presented with a total of 224 radio sources towards the SMC, 60 of which are classified here for the first time.

The radio spectral index $\alpha$ has been estimated for 422 sources in the field of the LMC and 162 towards the SMC. We have investigated the distribution of the spectral index over different types of sources and found that the spectral index cannot be the only criterion in distinguishing SNRs, $\mathrm{H}$ II regions and background sources.
We have compared our radio catalogues with a range of catalogues from other wavelengths such as X-ray, UV, $\mathrm{H} \alpha$, optical, IR and CO. Luminosity functions have been investigated for SNRs and $\mathrm{H}$ II regions in both Clouds. We have checked the consistency of our background source number in the field of the MCs with published $\log N-\log S$ counts.

In total, 209 discrete radio sources in the LMC and the 37 sources in the SMC are classified here to be either H II regions or SNRs. We have plotted the positions of these intrinsic sources (Figs. 11 and 12) to investigate the structure of both Clouds. These sources are believed to be tracers for the possible spiral structure in galaxies and we found some indication of a spiral structure in the LMC with the centre somewhere in 30 Doradus. The source distributions throughout the SMC are grouped into two regions: the main-body and the eastern wing of the SMC.

Acknowledgements. We thank D. Meinert, D. Goddard, J. Lequeux and J. Caswell for considerable support and comments in manuscript. 


\section{References}

Alvarez H., Aparici J., May J., 1987, A\&A 176, 25

Amy S.W., 1994, Proc. ASA 11(1), 83

Armand C., Deharveng L., Caplan J., 1992, A\&A 265, 504

Banas K.R., Hughes J.P., Bronfman L., Nyman L.-A., 1997, ApJ 480, 607

Bolton J.G., Butler P.W., 1975, Aust. J. Phys. Astrophys. Suppl. 34, 33

Bothun G.D., Thompson I.B., 1988, AJ 96, 877

Bruhweiler F.C., Klinglesmith D.A., Gull T.R., Sofia S., 1987, ApJ 317, 152

Caplan J., Deharveng L., 1985, A\&AS 62, 63

Caplan J., Deharveng L., 1986, A\&A 155, 297

Caplan J., Ye T., Deharveng L., Turtle A.J., Kennicutt R.C., 1996, A\&A 307, 403

Chin Y.-N., Henkel C., Milar T.J., et al., 1996, A\&A 312, 33

Chin Y.-N., Henkel C., Whiteoak J.B., et al., 1997, A\&A 317, 548

Chu Y.H., Kennicutt R.C., 1988, AJ 95(4), 1111

Chu Y.H., Low M.M.M., Garcia-Segura G., Wakker B., Kennicutt R.C., 1993, ApJ 414, 213

Chu Y.H., Kennicutt R.C., 1994, ApJ 425, 720

Chu Y.H., Wakker B., Low M.M.M., Garcia-Segura G., 1994, AJ 108, 1696

Chu Y.H., Dickel J.R., Staveley-Smith L., Osterberg J., Smith C.R., 1995a, AJ 109, 1729

Chu Y.H., Chang H.W., Su Y.L., Low M.M.M., 1995b, ApJ 450, 157

Chu Y.H., Kennicutt R.C., Snowden S.L., et al., 1997, PASP 109,554

Chu Y.H., 1997, AJ 113,181

Clarke J.N., Little A.G., Mills B.Y., 1976, Aust. J. Phys. Astrophys. Suppl. 40, 1

Cohen R., Dame T.M., Garay G., et al., 1988, ApJ 331, L95

Copetti M.V.F., Dottori H.A., 1989, A\&AS 77, 327

Cornett R.H., Hill J.K., Bohlin R.C., et al., 1994, ApJ 430, L117

Cornett R.H., Greason M.R., Hill J.K., et al., 1997, AJ 113, 1011

Corwin H.G., de Vaucouleurs A., de Vaucouleurs G., 1985, Southern Galaxy Catalogue, Austin

Davies R.D., Elliott K.H., Meaburn J., 1976, Mem. R. astr. Soc. 81,89

DeGioia-Eastwood K., 1992, ApJ 397, 542

Dennefeld M., 1982, A\&A 157, 267

Dickel J.R., Milne D.K., Junkes N., Klein U., 1993, A\&A 275, 265

Dickel J.R., Milne D.K., 1994, Proc. Astron. Soc. Australia 11, 99

Dickel J.R., Milne D.K., Kennicutt R.C., Chu Y.H., Schommer A., 1994, AJ 107(3), 1067

Dickel J.R., Chu Y.H., Gelino C., et al., 1995, ApJ 448, 623

Dickel J.R., Milne D.K., 1995, AJ 109, 200

Dickey J.M., Amy S., Haynes R.F., et al., 1994, A\&A 289, 357

Dopita M.A., Ford V.L., McGregor P.J., Mathewson D.S., Wilson I.R, 1981a, ApJ 250, 103

Dopita M.A., Tuohy I.R., Mathewson D.S., 1981b, ApJ 248, L105

Ellingsen S.P., Whiteoak J.B., Vaile R.A., 1994, MNRAS 269, 1019
Feitzinger J.V., Haynes R.F., Klein U., Wielebinski R. Perschke M., 1987, Vistas Astron. 30, 243

Filipović M.D., Haynes R.F., White G.L., et al., 1995, A\&AS 111, 311 (Paper IV)

Filipović M.D., White G.L., Haynes R.F., et al., 1996, A\&AS 120, 77 (Paper IVa)

Filipović M.D., Haynes R.F., White G.L., et al., 1997, A\&AS 121, 321 (Paper V)

Filipović M.D., Pietsch W.N., Haynes R.F., et al., 1998a, A\&AS 127, 119 (Paper VI)

Filipović M.D., Jones P.A., White G.L., Haynes R.F., 1998b, A\&AS (in press) (Paper VIII)

Fujimoto M., Kumai Y., 1991, in: Haynes R.F., Milne D.K., (eds.) Proc. IAU Symp. 148, The Magellanic Clouds. Reidel, Dordrecht, p. 469

Garay G., Rubio M., Ramirez S., Johansson L.E.B., Thaddeus P., 1993, A\&A 274, 743

Gilmozzi R., Murdin P., Clark D.H., Malin D., 1983, MNRAS 202, 927

Graham J.R., Evans A., Albinson J.S., Bode M.F., Meikle W.P.S., 1987, ApJ 319, 126

Gurwell M., Hodge P., 1990, PASP 102, 849

Haynes R.F., Klein U., Wielebinski R., Murray J.D., 1986, A\&A 159, 22

Haynes R.F., Klein U., Wayte S.R., et al., 1991, A\&A 252, 475

Henize K.G., 1956, ApJS 2, 315

Heydari-Malayeri M., Niemela V.S., Testor G., 1987, A\&A 184, 300

Heydari-Malayeri M., Lecavelier des Etangs A., 1994, A\&A 291, 960

Hodge P.W., Wright F.W., 1967, The Large Magellanic Cloud. Smithsonian press. Washington DC

Hodge P.W., Snow T.P., 1975, AJ 80, 9

Hodge P.W., Wright F.W., 1977, The Small Magellanic Cloud. University of Washington press. Seattle \& London

Hodge P.W., 1985, PASP 97, 530

Hughes P.J., et al., 1995, ApJ 444, L81

Hunt M.R., Whiteoak J.B., 1994, Proc. ASA 11, 68

Hunt M.R, PhD thesis, University of Western Sydney (in preparation)

Hwang U., Hughes J.P., Canizares C.R., Markert T.H., 1993, ApJ 414, 219

Inoue H., Koyama K., Tanaka Y., 1983, in: Danziger I.J., Gorenstein P. (eds.) Proc. IAU Symp. 101, Supernova Remnants and their X-Ray Emission. Reidel, Dordrecht, p. 535

Israel F.P., Johansson L.E.B., Lequeux J., et al., 1993, A\&A 276,25

Jones P.A., McAdam W.B., 1992, ApJS 80, 137

Jones T.J., Hyland A.R., Straw S., et al., 1986, MNRAS 219, 603

Kahabka P., Pietsch W., 1993, in: Baschek B., Klare G., Lequeux J. (eds.) Proc. of the Second European Meeting on the Magellanic Cloud 328, New Aspects of Magellanic Cloud Research. Reidel, Dordrecht, p. 71

Kahabka P., Pietsch W., 1996, A\&A 312, 919

Kahabka et al., 1997, A\&AS (in preparation)

Kennicutt R.C., Hodge P.W., 1986, ApJ 306, 130

Kennicutt R.C., 1994, in: Violent Star Formation from 30 Doradus to QSO's, Tenorio-Tagle G. (ed.). Cambridge University Press, Cambridge 
Kim S., Freeman K.C., Staveley-Smith L., et al., 1997, PASA 14,119

Klein U., Wielebinski R., Haynes R.F., Malin F.D., 1989, A\&A 211,280

Klein U., et al., 1991, in: Haynes R.F., Milne D.K. (eds.) Proc. IAU Symp. 148, The Magellanic Clouds. Reidel, Dordrecht, p. 31

Klein U., Haynes R.F., Wielebinski R., Meinert D., 1993, A\&A 271,402

Kutner M.L., Rubio M., Booth R.S., et al., 1997, A\&AS, 122, 255

Large M.I., Mills B.Y., Little A.G., Crawford D.F., Sutton J.M., 1981, MNRAS 194, 693

Laustsen S., Madsen C., West R.M., 1987, Exploring the Southern Sky. Springer-Verlag, Berlin

Laval A., Rosado M., Boulesteix J., et al., 1989, A\&A 208, 230

Laval A., Rosado M., Boulesteix J., et al., 1992, A\&A 253, 213

le Coarer E., Rosado M., Georgelin Y.P., Viale A., Goldes G., 1993, A\&A 280, 365

Loiseau N., Klein U., Greybe A., Wielebinski R., Haynes R. F., 1987, A\&A 178, 62

Long K.S., Helfand D.J., Grabelsky D.A., 1981, ApJ 248, 925

Lozinskaya T.A., 1992, Supernovae and Stellar Wind in the Interstellar Medium, American Institute of Physics, p. 255

Lucke P.B., Hodge P.W., 1970, AJ 75(2), 171

Luks T., Rohlfs K., 1992, A\&A 263, 41

Manchester R.N., Staveley-Smith L., Kesteven M.J., 1993, ApJ 411, 756

Martin N., Prevot L., Reberiot E., Rousseau J., 1976, A\&A 51, 31

Mathewson D.S., Healey J.R., 1964a, in: Kerr F.J., Rodgers A.W., (eds.), The Galaxy and the Magellanic Clouds. Canberra, Australian Academy of Science, p. 246

Mathewson D.S., Healey J.R., 1964b, in: Kerr F.J., Rodgers A.W. (eds.), The Galaxy and the Magellanic Clouds. Canberra, Australian Academy of Science, p. 283

Mathewson D.S., Ford V.L., Dopita M.A., et al., 1983, ApJS 51,345

Mathewson D.S., Ford V.L., Dopita M.A., et al., 1983a, in: Danziger I.J., Gorenstein P. (eds.) Proc. IAU Symp. 101, Supernova Remnants and their X- Ray Emission. Reidel, Dordrecht, p. 541

Mathewson D.S., Ford V.L., Dopita M.A., et al., 1984, ApJS 55,189

Mathewson D.S., Ford V.L., Tuohy I.R., et al., 1985, ApJS 58, 197

Mathewson D.S., Wayte S.R., Ford V.L., Ruan K., 1987, Proc. ASA 7, 19

McGee R.X., Brooks J.W., Batchelor R.A., 1972a, Aust. J. Phys. 25, 581

McGee R.X., Brooks J.W., Batchelor R.A., 1972b, Aust. J. Phys. 25, 613

McGee R.X., Newton L.M., 1972, Aust. J. Phys. 25, 619

McGee R.X., Newton L.M., Butler P.W., 1976, Aust. J. Phys. 29, 329

McGee R.X., Newton L.M., Butler P.W., 1978, MNRAS 183, 799

Meaburn J., Laspias V.N., 1991, A\&A 245, 635

Meinert D., 1992, PhD thesis, University of Bonn

Meurer G.R., Bicknell G.V., Gingold R.A., 1985, Proc. ASA 6,195
Mezger P.G., 1972, in Interstellar Matter. Saas Fee: Swiss Soc. of Astr. and Ap., p. 152 and 166

Mills B.Y., 1955, Aust J. Phys. Astrophys. 8, 368

Mills B.Y., Aller L.H., 1971, Aust J. Phys. 24, 609

Mills B.Y., Little A.G., 1953, Aust J. Phys. 6, 272

Mills B.Y., Little A.G., 1959, Handbuch für Physik, p. 239

Mills B.Y., Little A.G., Durdin J.M., Kesteven M.J., 1982, MNRAS 200, 1007

Mills B.Y., 1983, in: Danziger I.J., Gorenstein P. (eds.) Proc. IAU Symp. 101, Supernova Remnants and their X-Ray Emission. Reidel Dordrecht, p. 551

Mills B.Y., Turtle A.J., 1984a, in: Van den Bergh S., de Boer K.S. (eds.) Proc. IAU Symp. 108, Structure and evolution of the Magellanic Clouds. Reidel, Dordrecht, p. 283

Mills B.Y., Turtle A.J., Little A.G., Durdin M.J., 1984b, Aust. J. Phys. 37, 321

Milne D.K., Caswell J.L., Haynes R.F., 1980, MNRAS 191, 469

Morgan D.H., 1994, A\&AS 103, 235

Morgan D.H., 1995, A\&AS 112, 445

Mountfort P.I., Jonas J.L., de Jager G., Baart E.E., 1987, MNRAS 226, 917

Murai T., Fujimoto M., 1980, Pub. Astr. Soc. Japan 32, 581

Norci L., Ögelman H., 1995, A\&A 302, 879

Oey M.S., Massey P., 1995, ApJ 452, 210

Otrupcek R.E., Wright A.E., 1991, Proc. ASA 9, 170

Page T., Carruthers G.R., 1978, S201 Far-Ultraviolet Atlas of the LMC, Naval Research Lab. Washington

Pietsch W., Kahabka P., 1993, in: Baschek B., Klare G., Lequeux J. (eds.) Proc. of the Second European Meeting on the Magellanic Cloud 328, New Aspects of Magellanic Cloud Research. Reidel, Dordrecht, p. 59

Pietsch W., et al., A\&AS (in preparation)

Pizzichini G., Cline T.L., Desai U.D., et al., 1983, in: Danziger I.J., Gorenstein P. (eds.) Proc. IAU Symp. 101, Supernova Remnants and their X- Ray Emission. Reidel, Dordrecht, p. 573

Rosa M.R., 1993, in: Baschek B., Klare G., Lequeux J. (eds.) Proc. of the Second European Meeting on the Magellanic Cloud 328, New Aspect of Magellanic Cloud Research. Reidel, Dordrecht, p. 145

Rosado M., Laval A., le Coarer E., et al., 1993a, A\&A 272, 541

Rosado M., le Coarer E., Georgelin Y.P., Viale A., 1993b, in: Baschek B., Klare G., Lequeux J. (eds.) Proc. of the Second European Meeting on the Magellanic Cloud 328, New Aspect of Magellanic Cloud Research. Reidel, Dordrecht, p. 226

Rosado M., Le Coarer E., Georgelin Y.P., 1994, A\&A 286, 231

Rosado M., Laval A., le Coarer E., et al., 1996, A\&A 308, 588

Russel S.C., Dopita A.M., 1990, ApJS 74, 93

Rubio M., Garay G., Montani J., Thaddeus P., 1991, ApJ 368, 173

Rubio M., Lequeux J., Boulanger F., et al., 1993, A\&A 271, 1 Rubio M., Lequeux J., Boulanger F., et al., 1996, A\&AS 118, 263

Sabalisck H.S., Abraham Z., 1991, in: Haynes R.F., Milne D.K. (eds.) Proc. IAU Symp. 148, The Magellanic Clouds. Reidel, Dordrecht, p. 230

Sanduleak N., MacConnell D.J., Davis Philip A.G., 1978, PASP 90, 621

Sanduleak N., 1984, in: Van den Bergh S., de Boer K.S. (eds.) Proc. IAU Symp. 108, Structure and Evolution of the Magellanic Clouds. Reidel, Dordrecht, p. 231 
Savage A., 1976, MNRAS 174, 259

Savage A., Bolton J.G., Wright A.E., 1976, MNRAS 175, 517

Savage A., Bolton J.G., Wright A.E., 1977, MNRAS 179, 135

Schmidtke P.C., Cowley A.P., Frattare L.M., McGrath T.K. 1994, PASP 702, 843

Schmidt-Kaler T., 1993, in: Baschek B., Klare G., Lequeux J. (eds.) Proc. of the Second European Meeting on the Magellanic Cloud 328, New Aspect of Magellanic Cloud Research. Reidel, Dordrecht, p. 22

Schwering P.B.W., 1989, A\&AS 79, 79

Schwering P.B.W., Israel P.F., 1989, A\&AS 79, 105

Schwering P.B.W., Israel P.F., 1990, Atlas and Catalogue of Infrared Sources in the Magellanic Clouds. Kluwer, Dordrecht

Seward F.D., Mitchell M., 1981, ApJ 243, 736

Shain C.A., 1959, in: Bracewell R.N. (eds.) Paris Symposium on Radioastronomy. Stanford University Press, p. 328

Shapley H., 1956, Am. Sci. 44, 73

Shull P. Jr., 1983, ApJ 275, 592

Sinnott R.W., NGC 2000, 1988. Cambridge University Press, p. 46

Smith A.M., Cornett R.H., Hill R.S., 1987, ApJ 320, 609

Smith R.C., Chu Y.H., Low M.M.M., Oey M.S., Klein U., 1994, AJ 108, 1266

Snowden S. L., Petre R., 1994, ApJ 436, L123

Staveley-Smith L., Sault R.J., McConnell D., et al., 1995, Proc. ASA 12,13

Staveley-Smith L., Sault R.J., Hatzidimitriou D., Kesteven M.J., McConnell D., 1997, MNRAS 289, 225

Taylor J.H., Manchester R.N., Lyne A.G., 1993, ApJS 88, 529

Trümper J., Hasinger G., Aschenbach B., et al., 1991, Nat 349, 579

Tuohy I.R., Dopita M.A., Mathewson D.S., Long K.S., Helfand D.J., 1982, ApJ 261, 473

Tuohy I.R., Dopita M.A., Mathewson D.S., Long K.S., Helfand
D.J., 1983, in: Danziger I.J., Gorenstein P. (eds.) Proc. IAU Symp. 101, Supernova Remnants and their X-ray Emission. Reidel, Dordrecht, p. 559

Vancura O., Blair W.P., Long K.S., Raymond J.C., 1992, ApJ 394,158

Walborn N.R., Parker J.W., 1992, ApJ 399, L87

Wall J.V., 1994, Aust. J. Phys. 47, 625

Wang Q., Hamilton T., Helfand D.J., Wu X., 1991, ApJ 374, 475

Wang Q., Helfand D.J., 1991a, ApJ 370, 541

Wang Q., Helfand D.J., 1991b, ApJ 373, 497

Wang Q., Wu X., 1992, ApJS 78, 391

Westerlund B.E., 1993, in: Baschek B., Klare G., Lequeux J. (eds.) Proc. of the Second European Meeting on the Magellanic Cloud 328, New Aspects of Magellanic Cloud Research. Reidel, Dordrecht, p. 7

White G.L., Batty M.J., Bunton J.D., Brown D.R., Corben J.B., 1987, MNRAS 227, 705

White G.L., Bunton J.D., Anderson M.W.B., et al., 1991, MNRAS 248, 398

Wilcots E.M., 1994, AJ 107(4), 1338

Williams R.M., Chu Y.H., Dickel J.R., Beyer R., 1997, ApJ 480, 618

Wright A.E., Griffith M., Burke B., Ekers R.D., 1994, ApJS 91, 111

Xu C., Klein U., Meinert D., Wielebinski R., Haynes R.F., 1992, A\&A 257, 47

Ye T., 1988, PhD Thesis, Sydney University

Ye T., Turtle A.J., Kennicutt R.C., 1991, MNRAS 249, 722

Ye T., Turtle A.J., 1993, in: Baschek B., Klare G., Lequeux J. (eds.) Proc. of the Second European Meeting on the Magellanic Cloud 328, New Aspect of Magellanic Cloud Research. Reidel, Dordrecht, p. 168

Ye T., Amy S.W., Wang Q.D., Ball L., Dickel J., 1995, MNRAS 275,1218 